This is a post-peer-review, pre-copyedit version of an article published in Semigroup Forum. The final authenticated version is available online at: https://doi.org/10.1007/s00233-018-9931-8

\title{
CONGRUENCES ON DIRECT PRODUCTS OF TRANSFORMATION AND MATRIX MONOIDS
}

\author{
JOÃO ARAÚJO, WOLFRAM BENTZ, AND GRACINDA M.S. GOMES
}

\begin{abstract}
Mal'cev described the congruences of the monoid $\mathcal{T}_{n}$ of all full transformations on a finite set $X_{n}=\{1, \ldots, n\}$. Since then, congruences have been characterized in various other monoids of (partial) transformations on $X_{n}$, such as the symmetric inverse monoid $\mathcal{I}_{n}$ of all injective partial transformations, or the monoid $\mathcal{P} \mathcal{T}_{n}$ of all partial transformations.

The first aim of this paper is to describe the congruences of the direct products $Q_{m} \times P_{n}$, where $Q$ and $P$ belong to $\{\mathcal{T}, \mathcal{P} \mathcal{T}, \mathcal{I}\}$.

$\mathrm{Mal}^{\prime} \mathrm{cev}$ also provided a similar description of the congruences on the multiplicative monoid $F_{n}$ of all $n \times n$ matrices with entries in a field $F$; our second aim is to provide a description of the principal congruences of $F_{m} \times F_{n}$.

The paper finishes with some comments on the congruences of products of more than two transformation semigroups, and on a number of related open problems.
\end{abstract}

\section{INTRODUCTION}

Let $\mathcal{P} \mathcal{T}_{n}$ denote the monoid of all partial transformations on the set $X_{n}=\{1, \ldots, n\}$. Let $S_{n}$ denote the symmetric group on $X_{n}$. Let $\mathcal{T}_{n}$ be full transformation monoid, that is, the semigroup of all transformations in $\mathcal{P} \mathcal{T}_{n}$ with domain $X_{n}$; and let $\mathcal{I}_{n}$ be the symmetric inverse monoid, that is, the semigroup of all 1-1 maps contained in $\mathcal{P} \mathcal{T}_{n}$. The congruences of these semigroups were described in the past: $\mathrm{Mal}^{\prime} \mathrm{cev}$ [33] for $\mathcal{T}_{n}$, Šutov [38] for $\mathcal{P} \mathcal{T}_{n}$ and Liber [32] for $\mathcal{I}_{n}$.

In this paper we provide a description of the principal congruences of $Q_{n} \times Q_{m}$ (Theorem 3.11), where $Q \in\{\mathcal{P} \mathcal{T}, \mathcal{T}, \mathcal{I}\}$, and then use this result to provide the full description of all congruences of these semigroups (Theorem 4.11).

Similarly, for a field $F$, denote by $F_{n}$ the monoid of all $n \times n$ matrices with entries in $F$. The congruences of $F_{n}$ have been described by Mal'cev [34] (see also [27]). Here we provide a description of the principal congruences of $F_{n} \times F_{m}$ (Theorems 7.8, 7.10, 7.12, and 7.13).

It is worth pointing out that the descriptions of the congruences of the semigroups

$$
S:=\prod_{i \in M} Q_{i} \text { and } T:=\prod_{i \in M} F_{i},
$$

where $F$ is a field, $M$ is a finite multiset of natural numbers, and $Q \in\{\mathcal{P} \mathcal{T}, \mathcal{T}, \mathcal{I}\}$, are in fact yielded by the results of this paper, modulo the use of heavy notation and very long, but not very informative, statements of theorems. (For more details we refer the reader to Section 5.)

It is well known that the description of the congruence classes of a semigroup, contrary to what happens in a group or in a ring, poses special problems and usually requires very delicate considerations (see [26, Section 5.3]). Therefore it is no wonder that the study of congruences is among the topics attracting more attention when researching semigroups, something well illustrated by the fact that the few years of this century already witnessed the publication of more than 250 research papers on the topic.

Given the ubiquitous nature of the direct product construction, it comes quite as a surprise to realize that almost nothing is known about congruences on direct products of semigroups, even when the congruences on each factor of the product are known. Here we start that study trusting that this will be the first contribution in a long sequence of papers describing the congruences of direct products of transformation semigroups. Before closing this introduction

2010 Mathematics Subject Classification. 20M20,20M25, $20 \mathrm{M} 18$.

Key words and phrases. Monoid, congruences, Green relations. 
it is also worth to add that we have been led to this problem, not just by the inner appeal of a natural idea (describing the congruences of direct products of very important classes of semigroups whose congruences were already known), but by considerations on the centralizer in $\mathcal{T}_{n}$ of idempotent transformations. More about this will be said on the problems section at the end of the paper.

In order to outline the structure of the paper, we now introduce some notation. Let $S$ be a finite monoid. We say that $a, b \in S$ are $\mathcal{H}$-related if $a S=b S$ and $S a=S b$.

The elements $a, b \in S$ are said to be $\mathcal{D}$-related if $S a S=S b S$.

In Section 2, we recall the description of the congruences on $Q_{i}$, which we use in Section 3 to fully describe the principal congruences on $Q_{m} \times Q_{n}$, for $Q \in\{\mathcal{P} \mathcal{T}, \mathcal{T}, \mathcal{I}\}$. In Section 4, we show that a congruence $\theta$ on $Q_{m} \times Q_{n}$ is determined by those unions of its classes that are also unions of $\mathcal{D}$-classes, which we will call $\theta$-dlocks. After presenting the possible types of $\theta$-dlocks, whose properties are related to their $\mathcal{H}$-classes, we shall describe $\theta$ within each such block, making use of the results obtained in Section 3. In Section 5 we give an idea of how the congruences look like on a semigroup of the form $Q_{m} \times Q_{n} \times Q_{r}$. Section 6 is devoted to the description of the congruences on $F_{n}$ following [27], and we dedicate Section 7 to characterizing the principal congruences on $F_{m} \times F_{n}$. We do so following the pattern of Section 3, doing the necessary adaptations. Describing the general congruences of $F_{m} \times F_{n}$ is notationally heavy but we trust the reader will be convinced that to do so nothing but straightforward adaptions of Section 4 are needed. The paper finishes with a set of problems.

\section{Preliminaries}

For clarity, we start by recalling some well known facts on the Green relations as well as the description of the congruences on an arbitrary $Q_{m}$. The lattice of congruences of a semigroup $S$ will be denoted by $\operatorname{Con}(S)$. For further details see [24].

Given $f \in Q_{n}$ we denote its domain by $\operatorname{dom}(f)$, its image by $\operatorname{im}(f)$, its kernel by $\operatorname{ker}(f)$ and its rank (the size of the image of $f$ ) by $|f|$.

Lemma 2.1. Let $f, g \in Q_{n}$. Then

(1) $f \mathcal{D} g$ iff $|f|=|g|$;

(2) $f \mathcal{L} g$ iff $f$ and $g$ have the same image;

(3) $f \mathcal{R} g$ iff $f$ and $g$ have the same domain and kernel;

(4) $f \mathcal{H} g$ iff $f$ and $g$ have the same domain, kernel, and image.

Let $S$ be a monoid. A set $I \subseteq S$ is said to be an ideal of $S$ if $S I S \subseteq I$ and an ideal $I$ is said to be principal if there exists an element $a \in S$ such that $I=S a S$. It is well known that all ideals in $Q_{n}$ are principal; in fact, given any ideal $I \leq Q_{n}$, then $I=Q_{n} a Q_{n}$, for all transformation $a \in I$ of maximum rank. In addition we have $Q_{n} a Q_{n}=\left\{b \in Q_{n}|| b|\leq| a \mid\right\}$.

The Green relation $\mathcal{J}$ is defined on a monoid $S$ as follows: for $a, b \in S$,

$$
a \mathcal{J} b \text { iff } S a S=S b S .
$$

Thus two elements are $\mathcal{J}$-related if and only if they generate the same principal ideal. In a finite semigroup we have $\mathcal{J}=\mathcal{D}$ (and this explains the definition of $\mathcal{D}$ used in Section 1). It is easy to see that if $|f|<|g|$, then $S f S \subseteq S g S$ and in particular $f \in S g S$. That $f \in S g S$ implies $|f| \leq|g|$ is also obvious. Therefore $f \mathcal{J} g$ iff $|f|=|g|$.

If $f \mathcal{H} g$ then $f$ and $g$ have the same image, so we may speak about the set image im $H$ of an $\mathcal{H}$-class $H$. Given an $\mathcal{H}$-class $H$ of $Q_{n}$, we can fix an arbitrary linear order on im $H$, say that $\operatorname{im} f=\left\{a_{1}<\cdots<a_{|f|}\right\}$ for all $f \in H$. We define a right action - of the group $S_{i}$ with $i=|f|$ on all elements in $Q_{n}$ of rank $i$ : let $\omega \in S_{i}$ and $x \in \operatorname{dom}(f)$, then $(x) f \cdot \omega=a_{j \omega}$ where $x f=a_{j}$ with regard to the fixed ordering associated with the $\mathcal{H}$-class of $f$. Note that the action $\cdot$ preserves $\mathcal{H}$-classes. Hence for each $\omega \in S_{i}$ and $\mathcal{H}$-class $H$ with $|\operatorname{im} H|=i$, we may define $\bar{\omega}^{H} \in S_{\mathrm{im} H}$, where $S_{\operatorname{im} H}$ is the symmetric group over $\operatorname{im} H$, such that $(x) f \cdot \omega=((x) f) \bar{\omega}^{H}$, for all $f \in H$, $x \in \operatorname{im} H$.

The description of the congruences of $Q_{n}$ can be found in [24, sec. 6.3.15], and goes as follows. 
Theorem 2.2. A non-universal congruence of $Q_{n}$ is associated with a pair $(k, N)$, where $1 \leq$ $k \leq n$, and $N$ is a normal subgroup of $S_{k}$; and it is of the form $\theta(k, N)$ defined as follows: for all $f, g \in Q_{n}$,

$$
f \theta(k, N) g \text { iff }\left\{\begin{array}{l}
f=g \text { and }|f|>k, \text { or } \\
|f|,|g|<k, \text { or } \\
|f|=|g|=k, f \mathcal{H} g \text { and } f=g \cdot \omega, \text { where } \omega \in N .
\end{array}\right.
$$

We write $\theta=\theta(k, N)$ or just $\theta$ if there is no ambiguity. It follows from the normality of $N$ that the definition of $\theta(k, N)$ is independent of the ordering associated with each of the $\mathcal{H}$-classes of $Q_{n}$. A similar independence result will hold for a corresponding construction in our main result.

The following will be applied later without further reference. Let $g, g^{\prime} \in Q_{n}$. It follows from Theorem 2.2, that if $\left(g, g^{\prime}\right) \in \mathcal{H}$, then the principal congruence $\theta$ generated by $\left(g, g^{\prime}\right)$ is $\theta(|g|, N)$, where $N$ is the normal subgroup of $S_{|g|}$ generated by $\sigma \in S_{|g|}$ with $g^{\prime}=g \cdot \sigma$, with respect to a fixed ordering of the image of $g$. If $\left(g, g^{\prime}\right) \notin \mathcal{H}$ then $\theta$ is $\theta\left(k+1,\left\{\operatorname{id}_{S_{k+1}}\right\}\right)$, where $k=\max \left\{|g|,\left|g^{\prime}\right|\right\}$, or the universal congruence if $k=n$. In either case, this is the Rees congruence defined by the ideal $I_{k}$ of all transformations of rank less or equal to $k$, i.e. $\theta_{I_{k}}$.

From this description we see that if $\theta=\theta(k, N) \in \operatorname{Con}\left(Q_{n}\right)$ and there exist $f, g \in Q_{n}$, with $|f|<|g|$ and $(f, g) \in \theta$, then $|g|<k$ and the ideal generated by $g$ is contained in a single $\theta$-class.

For each $n>1$ the congruences on each semigroup $Q_{n}$ form a chain [24, sec. 6.5.1]. Let $\iota_{S}$ and $\omega_{S}$ be, respectively, the trivial and the universal congruences on $S$. For $k \in\{1, \ldots, n\}$, denote by $\equiv_{\varepsilon_{k}}$, $\equiv_{\mathcal{A}_{k}}$ and $\equiv_{S_{k}}$, the congruence associated to $k$ and to the trivial, the alternating and the symmetric subgroup of $S_{k}$, respectively. Finally, for $k=4$, let $\equiv_{V_{4}}$ be the congruence associated with the Klein 4-group. We have

$$
\begin{gathered}
\iota_{S}=\equiv_{S_{1}} \subset \equiv_{\varepsilon_{2}} \subset \equiv_{S_{2}} \subset \equiv_{\varepsilon_{3}} \subset \equiv_{\mathcal{A}_{3}} \subset \equiv_{S_{3}} \\
\subset \equiv_{\varepsilon_{4}} \subset \equiv_{V_{4}} \subset \equiv_{\mathcal{A}_{4}} \subset \equiv_{S_{4}} \subset \ldots \subset \equiv_{\varepsilon_{n}} \subset \equiv_{\mathcal{A}_{n}} \subset \equiv_{S_{n}} \subset \omega_{S} .
\end{gathered}
$$

Let $0 *$ stand for 1 if $Q=\mathcal{T}$ and for 0 in the other cases. For $0 * \leq i \leq m$, let $I_{i}^{(m)}$ stand for the ideal of $Q_{m}$ consisting of all functions $f$ with $|f| \leq i$. We will usually just write $I_{i}$ if $m$ is deducible from the context. Let $\theta_{I_{i}}$ stand for the Rees congruence on $Q_{m}$ defined by $I_{i}$.

\section{Principal Congruences on $Q_{m} \times Q_{n}$}

The aim of this section is to describe the principal congruences of $Q_{m} \times Q_{n}$, when $Q \in$ $\{\mathcal{I}, \mathcal{T}, \mathcal{P} \mathcal{T}\}$. We will start by transferring our notations to the setting of this product semigroup.

For functions $f \in Q_{m} \cup Q_{n}$, let $|f|$ once again stand for the size of the image of $f$, and for $(f, g) \in Q_{m} \times Q_{n}$ let $|(f, g)|=(|f|,|g|)$, where we order these pairs according to the partial order $\leq \times \leq$. Throughout, $\pi_{1}$ and $\pi_{2}$ denote the projections to the first and second factor.

We will start with some general lemmas about congruences on $Q_{m} \times Q_{n}$. In case there is no danger of ambiguity, we will use the shorthand $P$ for $Q_{m} \times Q_{n}$, to simplify the writing.

Lemma 3.1. Let $\theta$ be a congruence of $Q_{m} \times Q_{n}$ and fix $f \in Q_{m}$; let

$$
\theta_{f}:=\left\{\left(g, g^{\prime}\right) \in Q_{n} \times Q_{n} \mid(f, g) \theta\left(f, g^{\prime}\right)\right\} .
$$

Then

(1) $\theta_{f}$ is a congruence on $Q_{n}$;

(2) if $f^{\prime} \in Q_{m}$ and $\left|f^{\prime}\right| \leq|f|$, then $\theta_{f} \subseteq \theta_{f^{\prime}}$;

(3) if $|f|=\left|f^{\prime}\right|$ in $Q_{m}$, then $\theta_{f}=\theta_{f^{\prime}}$.

Proof. (1) That $\theta_{f}$ is an equivalence on $Q_{n}$ is clear. The compatibility follows from the fact that $Q_{m}$ has an identity; indeed

$$
\left(g, g^{\prime}\right) \in \theta_{f} \Rightarrow(f, g) \theta\left(f, g^{\prime}\right) \Rightarrow(f, g)(1, h) \theta\left(f, g^{\prime}\right)(1, h) \Rightarrow(f, g h) \theta\left(f, g^{\prime} h\right) \Rightarrow g h \theta_{f} g^{\prime} h .
$$

Similarly we prove the left compatibility. 
(2) If $\left|f^{\prime}\right| \leq|f|$ then $f^{\prime} \in Q_{m} f Q_{m}$ and hence we have $f^{\prime}=h f h^{\prime}$, for some $h, h^{\prime} \in Q_{m}$; in addition $(f, g) \theta\left(f, g^{\prime}\right)$ implies

$$
\left(f^{\prime}, g\right)=(h, 1)(f, g)\left(h^{\prime}, 1\right) \theta(h, 1)\left(f, g^{\prime}\right)\left(h^{\prime}, 1\right)=\left(f^{\prime}, g^{\prime}\right)
$$

so that $\theta_{f} \subseteq \theta_{f^{\prime}}$. Condition (3) follows from (2) and its symmetric.

In a similar way, given a congruence $\theta$ on $Q_{m} \times Q_{n}$ and fixed $g \in Q_{n}$, we define $\theta_{g}:=\left\{\left(f, f^{\prime}\right) \in\right.$ $\left.Q_{m} \times Q_{m} \mid(f, g) \theta\left(f^{\prime}, g\right)\right\}$.

The next result describes the ideals of $Q_{n} \times Q_{m}$.

Lemma 3.2. Let $Q \in\{\mathcal{I}, \mathcal{T}, \mathcal{P} \mathcal{T}\}$. The ideals of $P:=Q_{m} \times Q_{n}$ are exactly the unions of sets of the form $I_{i}^{(m)} \times I_{j}^{(n)}$, where $I_{i}^{(m)}$ and $I_{j}^{(n)}$ are ideals of $Q_{m}$ and $Q_{n}$, respectively.

Proof. That any union of sets of the form $I_{i}^{(m)} \times I_{j}^{(n)}$ is an ideal of $P$ is obvious.

Conversely, let $I$ be an ideal of $P$, and $(f, g) \in I$. It is self-evident that $(f, g) \in I_{|f|} \times I_{|g|}$. Then, by the definition of an ideal of $P$, we have $P(f, g) P \subseteq I$, for every $(f, g) \in I$. Let $\left(f^{\prime}, g^{\prime}\right) \in I_{|f|} \times I_{|g|}$. Then $f^{\prime} \in Q_{m} f Q_{m}$ and $g^{\prime} \in Q_{n} g Q_{n}$ so that $\left(f^{\prime}, g^{\prime}\right) \in P(f, g) P \subseteq I$. It follows that $\cup_{(f, g) \in I} I_{|f|} \times I_{|g|} \subseteq I$. Regarding the reverse inclusion, let $(f, g) \in I$; then $\cup_{(f, g) \in I} I_{|f|} \times I_{|g|} \supseteq I$. The result follows.

Lemma 3.3. Let $\theta$ be a congruence of $P:=Q_{m} \times Q_{n}$.

(1) If $Q \in\{\mathcal{P} \mathcal{T}, \mathcal{I}\}$, then $\theta$ contains a class $I_{\theta}$ which is an ideal;

(2) If $Q=\mathcal{T}$ and both $\pi_{1}(\theta)$ and $\pi_{2}(\theta)$ are non-trivial, then $\theta$ contains a class $I_{\theta}$ which is an ideal;

(3) $\theta$ contains at most one ideal class.

Proof. (1) If $Q$ is $\mathcal{P} \mathcal{T}$ or $\mathcal{I}$, then $P$ has a zero, whose congruence class is easily seen to be a unique ideal of $P$.

(2) If $Q$ is $\mathcal{T}$, let $c_{a}$ denote the constant map whose image is $\{a\}$, for some $a \in\{1, \ldots, m\}$. Since $\pi_{2}(\theta)$ is non-trivial, it follows that there exist $f, f^{\prime} \in Q_{m}$ and distinct $g, g^{\prime} \in Q_{n}$ such that $(f, g) \theta\left(f^{\prime}, g^{\prime}\right)$. Thus $(f, g)\left(c_{a}, 1\right) \theta\left(f^{\prime}, g^{\prime}\right)\left(c_{a}, 1\right)$, that is, $\left(c_{a}, g\right) \theta\left(c_{a}, g^{\prime}\right)$, so that $\theta_{c_{a}}$ is non-trivial. By Theorem 2.2, the ideal $I_{1}^{(n)}$ is contained in a class of $\theta_{c_{a}}$. Similarly, we pick $b \in\{1, \ldots, n\}$ concluding that $I_{1}^{(m)} \times\left\{c_{b}\right\}$ lies in a $\theta$-class.

We claim that $I_{1}^{(m)} \times I_{1}^{(n)}$ is contained in one $\theta$-class. In fact, let $\left(c_{a}, c_{b}\right),\left(c_{d}, c_{e}\right) \in I_{1}^{(m)} \times I_{1}^{(n)}$. Then $\left(c_{a}, c_{d}\right) \in \theta_{c_{e}}$, since $I_{1}^{(m)} \times\left\{c_{e}\right\}$ lies in a $\theta$-class, and similarly $\left(c_{b}, c_{e}\right) \in \theta_{c_{a}}$. Thus $\left(c_{a}, c_{e}\right) \theta\left(c_{a}, c_{b}\right)$ and $\left(c_{d}, c_{e}\right) \theta\left(c_{a}, c_{e}\right)$. Therefore by transitivity we get $\left(c_{a}, c_{b}\right) \theta\left(c_{d}, c_{e}\right)$. We have proved that $I_{1}^{(m)} \times I_{1}^{(n)}$ is contained in the $\theta$-class of $\left(c_{a}, c_{b}\right)$.

Conversely, given any $(f, g)$ in the $\theta$-class of $\left(c_{a}, c_{b}\right)$ and any $\left(f^{\prime}, g^{\prime}\right) \in P$, we have

$$
\left(f f^{\prime}, g g^{\prime}\right) \theta\left(c_{a} f^{\prime}, c_{b} g^{\prime}\right) \text { and }\left(c_{a} f^{\prime}, c_{b} g^{\prime}\right) \in I_{1}^{(m)} \times I_{1}^{(n)} \subseteq\left[\left(c_{a}, c_{b}\right)\right]_{\theta},
$$

so $\left(f f^{\prime}, g g^{\prime}\right) \in\left[\left(c_{a}, c_{b}\right)\right]_{\theta}$; similarly we prove that $\left(f^{\prime} f, g^{\prime} g\right) \in\left[\left(c_{a}, c_{b}\right)\right]_{\theta}$. Thus $\left[\left(c_{a}, c_{b}\right)\right]_{\theta}$ is an ideal.

(3) The last assertion holds in all semigroups, as any ideal class is a (necessarily unique) zero element of the quotient semigroup $P / \theta$.

For the remainder of this section we fix the following notations. Let $Q \in\{\mathcal{T}, \mathcal{P} \mathcal{T}, \mathcal{I}\}, f, f^{\prime} \in$ $Q_{m}$ and $g, g^{\prime} \in Q_{n}$. Let $\theta$ be a principal congruence on $Q_{m} \times Q_{n}$ generated by $\left((f, g),\left(f^{\prime}, g^{\prime}\right)\right)$. Let $\theta_{1}$ be the principal congruence generated by $\left(f, f^{\prime}\right)$ in $Q_{m}$ and $\theta_{2}$ be the principal congruence generated by $\left(g, g^{\prime}\right)$ in $Q_{n}$.

Lemma 3.4. If $f=f^{\prime}$ and $g \neq g^{\prime}$, we have $(a, b) \theta(c, d)$ if and only if $(a, b)=(c, d)$ or $a=c$, $|a| \leq|f|$ and $b \theta_{2} d$.

Proof. Let $\theta^{\prime}$ be the binary relation defined by the statement of the lemma. If $|a| \leq|f|$, then $a=u f v$, for some $u, v \in Q_{m}$ and hence $\left((a, g),\left(a, g^{\prime}\right)\right)=\left((u f v, 1 g 1),\left(u f^{\prime} v, 1 g^{\prime} 1\right)\right) \in \theta$. It follows that $\left(g, g^{\prime}\right) \in \theta_{a}$ whence $\theta_{2} \subseteq \theta_{a}$. Now if $(b, d) \in \theta_{2}$, then $(b, d) \in \theta_{a}$, and therefore $(a, b) \theta(a, d)$. Hence $\theta^{\prime} \subseteq \theta$. 
Conversely, it is straightforward to check that $\theta^{\prime}$ is a congruence containing $\left((f, g),\left(f, g^{\prime}\right)\right)$, thus $\theta \subseteq \theta^{\prime}$. The result follows.

The following corollary gives a more direct description of the congruences covered by Lemma 3.4 by incorporating the structure of the congruence $\theta_{2}$ on $Q_{n}$. By applying Theorem 2.2, we obtain

Corollary 3.5. If $\left(g, g^{\prime}\right) \notin \mathcal{H}$, let $k=\max \left\{|g|,\left|g^{\prime}\right|\right\}$. Then $\theta_{2}$ is the Rees congruence $\theta_{I_{k}}$ and $(a, b) \theta(c, d)$ if and only if one of the following holds:

(1) $(a, b)=(c, d),|a|=|c|>|f|$ or $|b|=|d|>k$;

(2) $a=c$ and $|a|=|c| \leq|f|,|b|,|d| \leq k$.

If $\left(g, g^{\prime}\right) \in \mathcal{H}$ and $g \neq g^{\prime}$ then $\theta_{2}=\theta(k, N)$, for $k=|g|$ and $N$ is the normal subgroup of $S_{k}$ generated by $\sigma$, where $g^{\prime}=g \cdot \sigma$. Moreover, $(a, b) \theta(c, d)$ if and only if one of the following holds:

(1) $(a, b)=(c, d),|a|=|c|>|f|$ or $|b|=|d|>k$;

(2) $a=c,|b|=|d|=k, b \mathcal{H} d$ and $d=b \cdot \omega$ for some $\omega \in N$;

(3) $a=c,|a|=|c| \leq|f|,|b|,|d|<|g|$.

Lemma 3.6. Let $j=\max \left\{|f|,\left|f^{\prime}\right|\right\}$. If $g \neq g^{\prime}$ and $\left(f, f^{\prime}\right) \notin \mathcal{H}$, then $\theta_{g}$ or $\theta_{g^{\prime}}$ contains the Rees congruence $\theta_{I_{j}}$ of $Q_{m}$.

Proof. We will show that for $j=\left|f^{\prime}\right|, \theta_{I_{j}} \subseteq \theta_{g^{\prime}}$. An analogous result for $j=|f|$ follows symmetrically. So let us assume that $|f| \leq\left|f^{\prime}\right|=j$. As $\left(f, f^{\prime}\right) \notin \mathcal{H}, f$ and $f^{\prime}$ must differ in either image or kernel. We consider two cases.

First case: $\operatorname{im} f \neq \operatorname{im} f^{\prime}$.

As $\left|f^{\prime}\right|=j \geq|f|, \operatorname{im} f^{\prime} \nsubseteq \mathbb{i m} f$. As $Q_{m}$ is regular, there exists an idempotent $h \in Q_{m}$ such that $h \mathcal{L} f$. Hence $\operatorname{im} h=\operatorname{im} f$, and as $h$ is idempotent, $f h=f$.

We have that $\left(f^{\prime} h, g^{\prime}\right)=\left(f^{\prime}, g^{\prime}\right)(h, 1) \theta(f, g)(h, 1)=(f h, g)=(f, g) \theta\left(f^{\prime}, g^{\prime}\right)$ and therefore $\left(f^{\prime} h, f^{\prime}\right) \in \theta_{g^{\prime}}$. As $\operatorname{im} f^{\prime} \nsubseteq \operatorname{im} f$, and $\operatorname{im} h=\operatorname{im} f$, the maps $f^{\prime} h$ and $f^{\prime}$ have different images. It follows that $\left(f^{\prime} h, f^{\prime}\right) \notin \mathcal{H}$. Now, the congruence $\theta^{\prime}$ generated by $\left(f^{\prime} h, f^{\prime}\right)$ is contained in $\theta_{g^{\prime}}$ and by Theorem 2.2, we have $\theta^{\prime}=\theta_{I_{j}}$. We get $\theta_{I_{j}} \subseteq \theta_{g^{\prime}}$.

Second case: $\operatorname{ker} f \neq \operatorname{ker} f^{\prime}$.

Now $\left|f^{\prime}\right|=j \geq|f|$, implies that $\operatorname{ker} f \nsubseteq \operatorname{ker} f^{\prime}$. As above, the regularity of $Q_{m}$ implies that there exists an idempotent $h$ that is $\mathcal{R}$-related to $f$; thus $h$ and $f$ have the same kernel. Hence

$$
\left(h f^{\prime}, g^{\prime}\right)=(h, 1)\left(f^{\prime}, g^{\prime}\right) \theta(h, 1)(f, g)=(h f, g)=(f, g) \theta\left(f^{\prime}, g^{\prime}\right)
$$

and so $\left(h f^{\prime}, f^{\prime}\right) \in \theta_{g^{\prime}}$. Now $\operatorname{ker} f=\operatorname{ker} h \subseteq \operatorname{ker}\left(h f^{\prime}\right)$. As $\operatorname{ker} f \nsubseteq \operatorname{ker} f^{\prime},\left(h f^{\prime}, f^{\prime}\right) \notin \mathcal{H}$. As above, by Theorem 2.2 , we get $\theta_{I_{\left|f^{\prime}\right|}}=\theta_{I_{j}} \subseteq \theta_{g^{\prime}}$.

Theorem 3.7. Let $\theta$ be the congruence on $Q_{m} \times Q_{n}$ generated by $\left((f, g),\left(f^{\prime}, g^{\prime}\right)\right)$, and assume that $\left(f, f^{\prime}\right) \notin \mathcal{H},\left(g, g^{\prime}\right) \notin \mathcal{H},|f|=i,\left|f^{\prime}\right|=j,|g|=k,\left|g^{\prime}\right|=l$. Then $\theta$ is the Rees congruence on $Q_{m} \times Q_{n}$ defined by the ideal $I=I_{i} \times I_{k} \cup I_{j} \times I_{l}$.

Proof. If $Q=\mathcal{T}$, then pick two arbitrary constants $z=c_{a}$ and $z^{\prime}=c_{b}$ in $Q_{m}$ and $Q_{n}$, respectively. If $Q \in\{\mathcal{P} \mathcal{T}, \mathcal{I}\}$, let $z, z^{\prime}$ be the empty maps in $Q_{m}$ and $Q_{n}$.

As $f \neq f^{\prime}$ and $g \neq g^{\prime}$, by Lemma 3.3, the congruence $\theta$ contains an ideal class $K$. As $\left(z, z^{\prime}\right)$ lies in the smallest ideal $I_{0 *} \times I_{0 *}$ of $P,\left(z, z^{\prime}\right) \in K$. We claim that $(f, g) \in K$.

To show this, note that by Lemma 3.6, either $\theta_{g}$ or $\theta_{g^{\prime}}$ contains the Rees congruence $\theta_{I_{\max \{i, j\}}}$.

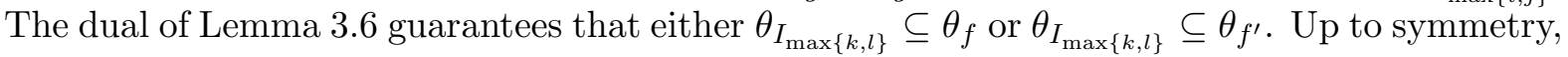
there are two cases.

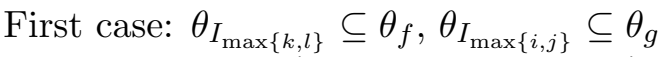

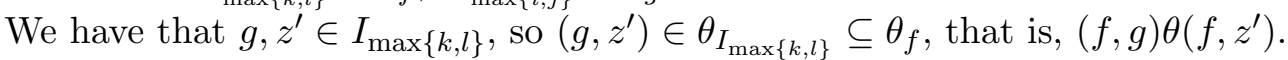

As $f, z \in I_{\max \{i, j\}}$, an analogous argument shows that $(f, z) \in \theta_{g}$. By Lemma 3.1, we have $\theta_{g} \subseteq \theta_{z^{\prime}}$, and so $(f, z) \in \theta_{z^{\prime}}$. Thus $\left(f, z^{\prime}\right) \theta\left(z, z^{\prime}\right)$ and hence $(f, g) \theta\left(f, z^{\prime}\right) \theta\left(z, z^{\prime}\right) \in K$.

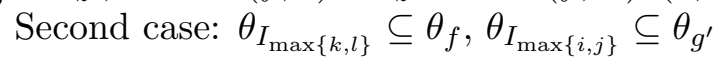

We have $g, z^{\prime} \in I_{\max \{k, l\}}$ so $\left(g, z^{\prime}\right) \in \theta_{f}$ and similarly $\left(f^{\prime}, z\right) \in \theta_{g^{\prime}}$. Thus

$$
\left(f, z^{\prime}\right) \theta(f, g) \theta\left(f^{\prime}, g^{\prime}\right) \theta\left(z, g^{\prime}\right) \text {. }
$$


Let $h \in S_{m}$ be such that $f h f=f$ (such $h$ clearly exists). We then have $(f, g) \theta\left(f, z^{\prime}\right)=\left(f h f, z^{\prime}\right)=\left(f, z^{\prime}\right)\left(h, z^{\prime}\right)\left(f, z^{\prime}\right) \theta\left(f, z^{\prime}\right)\left(h, z^{\prime}\right)\left(z, g^{\prime}\right)=\left(z, z^{\prime}\right) \in I_{0 *} \times I_{0 *} \subseteq K$.

Hence in both cases $(f, g) \in K$. As $K$ is a class of $\theta$, then $\left(f^{\prime}, g^{\prime}\right) \in K$ as well. Now $I \subseteq K$, as $I$ is the smallest ideal containing $\left\{(f, g),\left(f^{\prime}, g^{\prime}\right)\right\}$. It follows that $\theta_{I} \subseteq \theta_{K} \subseteq \theta$. Conversely $\theta \subseteq \theta_{I}$ as $\theta$ is generated by $\left((f, g),\left(f^{\prime}, g^{\prime}\right)\right)$ and $\theta_{I}$ is a congruence that contains $\left((f, g),\left(f^{\prime}, g^{\prime}\right)\right)$. Hence $\theta=\theta_{I}$.

Corollary 3.8. Under the conditions of Theorem 3.7, if $i \leq j$ and $k \leq l$ then $\theta=\theta_{I_{j} \times I_{l}}$.

Theorem 3.9. Let $\theta$ be the congruence on $Q_{m} \times Q_{n}$ generated by $\left((f, g),\left(f^{\prime}, g^{\prime}\right)\right)$, and let $\theta_{2}$ be the congruence on $Q_{n}$ generated by $\left(g, g^{\prime}\right)$. If $g \neq g^{\prime},\left(g, g^{\prime}\right) \in \mathcal{H}$ and $\left(f, f^{\prime}\right) \notin \mathcal{H}$; let $j=\max \left\{|f|,\left|f^{\prime}\right|\right\}$ and $k=|g|=\left|g^{\prime}\right|$. Then $(a, b) \theta(c, d)$ if and only if $(a, b)=(c, d)$ or $|a|,|c| \leq j$, $|b|,|d| \leq k, b \theta_{2} d$.

Proof. We start with some considerations having in mind the initial conditions. By the dual of Lemma 3.1, we have $\theta_{g}=\theta_{g^{\prime}} \subseteq \theta_{h}$ for all $h \in Q_{n}$ with $|h| \leq k$. By Lemma 3.6, we get $\theta_{I_{j}} \subseteq \theta_{g}=\theta_{g^{\prime}}$, hence that $\theta_{I_{j}} \subseteq \theta_{h}$ for each such $h$. Assume w.l.o.g. that $\left|f^{\prime}\right|=j$. Now $f, f^{\prime} \in I_{j}$ and $\theta_{I_{j}} \subseteq \theta_{g}$, so $f \theta_{g} f^{\prime}$. Thus $\left(f^{\prime}, g^{\prime}\right) \theta(f, g) \theta\left(f^{\prime}, g\right)$. Then $\left(g, g^{\prime}\right) \in \theta_{f^{\prime}}$ and therefore $\theta_{2} \subseteq \theta_{f^{\prime}}$, as $\theta_{2}$ is the congruence generated by $\left(g, g^{\prime}\right)$. By Lemma 3.1, $\theta_{f^{\prime}} \subseteq \theta_{u}$, for all $u \in Q_{m}$ such that $|u| \leq\left|f^{\prime}\right|=j$. Thus $\theta_{2} \subseteq \theta_{u}$ for all $u \in Q_{m}$ with $|u| \leq j$.

Let $\theta^{\prime}$ be the relation on $Q_{m} \times Q_{n}$ defined by $(a, b) \theta^{\prime}(c, d)$ if and only if $(a, b)=(c, d)$ or $|a|,|c| \leq j,|b|,|d| \leq k, b \theta_{2} d$. We want to show that $\theta=\theta^{\prime}$.

Assume that $|a|,|c| \leq j,|b|,|d| \leq k$ and $b \theta_{2} d$. Taking $u=a$ we obtain $\theta_{2} \subseteq \theta_{a}$. As $b \theta_{2} d$, we get $(a, b) \theta(a, d)$. Now $|d| \leq k$ which, as mentioned at the beginning of the proof, implies that $\theta_{I_{j}} \subseteq \theta_{d}$. It follows that $(a, c) \in \theta_{d}$, that is $(a, d) \theta(c, d)$. Therefore $(a, b) \theta(a, d) \theta(c, d)$, and so $\theta^{\prime} \subseteq \theta$.

For the reverse inclusion, it suffices to check that $\theta^{\prime}$ is a congruence containing $\left((f, g),\left(f^{\prime}, g^{\prime}\right)\right)$. We leave this straightforward verification to the reader.

Notice that we can once again give a more explicit description of $\theta$ by incorporating the classification of $\theta_{2}$ given by Theorem 2.2.

Corollary 3.10. Let $(f, g),\left(f^{\prime}, g^{\prime}\right) \in Q_{m} \times Q_{n}$, such that $g \neq g^{\prime},\left(g, g^{\prime}\right) \in \mathcal{H}$ and $\left(f, f^{\prime}\right) \notin \mathcal{H}$. Let $j=\max \left\{|f|,\left|f^{\prime}\right|\right\}$ and $k=|g|=\left|g^{\prime}\right|$. Let $g^{\prime}=g \cdot \sigma$ for $\sigma \in S_{k}$ with regard to some ordering associated with the $\mathcal{H}$-class of $g$, and let $N$ be the normal subgroup of $S_{k}$ generated by $\sigma$. If $\theta$ is the congruence on $Q_{m} \times Q_{n}$ generated by $\left((f, g),\left(f^{\prime}, g^{\prime}\right)\right)$, then $(a, b) \theta(c, d)$ if and only if one of the following holds:

(1) $(a, b)=(c, d)$ for $|a|>j$ or $|b|>k$;

(2) $|a|,|c| \leq j,|b|=k, b \mathcal{H} d$ and $d=b \cdot \omega$ for some $\omega \in N$, and with regard to some ordering associated with the $\mathcal{H}$-class of $d$;

(3) $|a|,|c| \leq j$ and $|b|,|d|<k$.

We remark that there are obvious dual versions of Lemma 3.4 and Theorem 3.9 obtained by switching the roles of the coordinates. Apart from the trivial case that $(f, g)=\left(f^{\prime}, g^{\prime}\right)$, it remains to determine the principal congruence $\theta$ when $f \neq f^{\prime}, g \neq g^{\prime}, f \mathcal{H} f^{\prime}, g \mathcal{H} g^{\prime}$.

We will first extend the actions - of $S_{i}$ on $Q_{m}$ and of $S_{j}$ on $Q_{n}$ to a partial action of $S_{i} \times S_{j}$ on $Q_{m} \times Q_{n}$. We define the action $\cdot$ of $S_{i} \times S_{j}$ on the set

$$
D_{i, j}=\left\{(f, g) \in Q_{m} \times Q_{n} \text { such that }|f|=i,|g|=j\right\}
$$

by setting $(f, g) \cdot\left(\omega_{1}, \omega_{2}\right)=\left(f \cdot \omega_{1}, g \cdot \omega_{2}\right)$, for all $(f, g) \in D_{i, j}$ and $\left(\omega_{1}, \omega_{2}\right) \in S_{i} \times S_{j}$, where in the first component - is applied with respect to the ordering of the $\mathcal{H}$-class of $f$ within $Q_{m}$, and correspondingly in the second component.

As $\mathcal{H}$-classes of $Q_{m} \times Q_{n}$ are products of $\mathcal{H}$-classes of $Q_{m}$ and of $Q_{n}$, it follows that the action $\cdot$ preserves $\mathcal{H}$-classes. In addition, the action - is transitive on each $\mathcal{H}$-class. If $H_{f}$ and $H_{g}$ stand for the $\mathcal{H}$-classes of $f$ in $Q_{m}$ and of $g$ in $Q_{n}$, we have $(f, g) \cdot\left(\omega_{1}, \omega_{2}\right)=\left(f \bar{\omega}_{1}^{H_{f}}, f \bar{\omega}_{2}^{H_{g}}\right)$, 
where $\bar{\omega}_{1}^{H_{f}} \in S_{\mathrm{im} f}$ and $\bar{\omega}_{2}^{H_{g}} \in S_{\mathrm{im} g}$ are as defined before Theorem 2.2. In this context, we will always consider $S_{\operatorname{im} f} \times S_{\operatorname{im} g}$ to be a subgroup of $S_{m} \times S_{n}$ in the natural way.

Theorem 3.11. Let $\theta$ be the principal congruence on $Q_{m} \times Q_{n}$ generated by $\left((f, g),\left(f^{\prime}, g^{\prime}\right)\right)$. If $f \neq f^{\prime}, g \neq g^{\prime}, f \mathcal{H} f^{\prime}, g \mathcal{H} g^{\prime},|f|=i=\left|f^{\prime}\right|,|g|=k=\left|g^{\prime}\right|$, let $\sigma_{1} \in S_{i}$ and $\sigma_{2} \in S_{k}$ be such that $f \cdot \sigma_{1}=f^{\prime}$ and $g \cdot \sigma_{2}=g^{\prime}$. Let $N$ be the normal subgroup of $S_{i} \times S_{k}$ generated by the pair $\left(\sigma_{1}, \sigma_{2}\right)$.

Then $(a, b) \theta(c, d)$ if and only $(a, b)=(c, d)$ or one of the following hold:

(1) $|a|,|c| \leq i-1,|b|,|d| \leq k-1$;

(2) $|a|=|c|=i,|b|,|d| \leq k-1$, and $a \theta_{1} c$;

(3) $|a|,|c| \leq i-1,|b|,|d|=k$, and $b \theta_{2} d$;

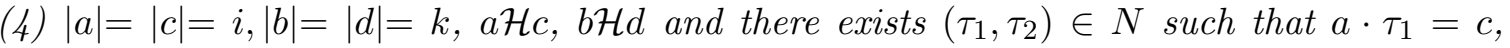
$b \cdot \tau_{2}=d$.

Proof. First note that if $(a, b) \theta(c, d),(a, b) \neq(c, d)$, and $\theta$ is generated by $\left((f, g),\left(f^{\prime}, g^{\prime}\right)\right)$, we must have $|a| \leq|f|=i,|b| \leq|g|=k,|c| \leq\left|f^{\prime}\right|=i,|d| \leq\left|g^{\prime}\right|=k$.

As $f \mathcal{H} f^{\prime}$, we have that $f$ and $f^{\prime}$ have the same kernel and image. Together with $f \neq f^{\prime}$, this implies $|f|=i \geq 2$. Similarly $k \geq 2$. Let $\operatorname{ker} f=\operatorname{ker} f^{\prime}=\left\{K_{1}, K_{2}, \ldots, K_{i}\right\}$.

First assume that $Q \in\{\mathcal{T}, \mathcal{P} \mathcal{T}\}$.

Suppose that $i \geq 3$. As $f \neq f^{\prime}$, there exists $x \in \operatorname{im} f=\operatorname{im} f^{\prime}$ such that the associated kernel classes are different, i.e. $f^{-1}(x) \neq f^{\prime-1}(x)$. W.l.o.g. assume $K_{1}=f^{-1}(x)$ and $K_{2}=f^{\prime-1}(x)$. Let $\{y\}=K_{3} f$ so that $f^{-1}(y)=K_{3}$.

Let $h \in \mathcal{T}_{n}$ be such that $y h=x$ and it is identical otherwise. Then $(f h, g) \theta\left(f^{\prime} h, g^{\prime}\right)$, where $|f h|=\left|f^{\prime} h\right|=i-1$ and $f h$ and $f^{\prime} h$ have different kernel, since the preimages of $x$ are $K_{1} \dot{\cup} K_{3}$ and $K_{2} \cup K_{j}$, for some $j$, respectively.

Let $\beta$ be the congruence generated by the pair $\left((f h, g),\left(f^{\prime} h, g^{\prime}\right)\right)$. As $\left(f h, f^{\prime} h\right) \notin \mathcal{H}, g \neq$ $g^{\prime}, g \mathcal{H} g^{\prime}$, Theorem 3.9 is applicable to $\beta$. Thus $(a, b) \beta(c, d)$ for $(a, b) \neq(c, d)$ if and only if $|a|,|c| \leq i-1,|b|,|d| \leq k$, and $b \theta_{2, \beta} d$, where $\theta_{2, \beta}$ is the $Q_{n}$-congruence generated by $\left(g, g^{\prime}\right)$ and hence is equal to $\theta_{2}$. By Theorem 2.2, the relation $\theta_{2}$ restricted to $I_{k-1}$ is the universal relation. Therefore the pairs $((a, b),(c, d))$ that satisfy condition (1) or $(3)$ are in $\beta$, but $\beta$ 's generating pair is in $\theta$, and so they are in $\theta$, as well.

Suppose now that $i=2$. Let $\left(K_{1}\right) f=x_{1}$ and $\left(K_{2}\right) f=x_{2}$. Then $\left(K_{1}\right) f^{\prime}=x_{2}$, and $\left(K_{2}\right) f^{\prime}=x_{1}$. Let $h$ be a total map with image contained in $K_{1}$. Then $(h f, g) \theta\left(h f^{\prime}, g^{\prime}\right)$ where $|h f|=\left|h f^{\prime}\right|=1$, but $\operatorname{im} h f \neq \operatorname{im} h f^{\prime}$. Thus $\left(h f, h f^{\prime}\right) \notin \mathcal{H}$, and by Theorem 3.9, as before, we conclude that $\theta$ must contain all pairs satisfying conditions (1) or (3).

Next suppose that $Q=\mathcal{I}$. We have $i \geq 2$. In this case, there exists $x \in \operatorname{im} f=\operatorname{im} f^{\prime}$ such that $f^{-1}(x) \neq f^{\prime-1}(x)$ (notice that these sets are now singletons). Let $h \in \mathcal{I}_{m}$ be the identity map with domain $\{1, \ldots, m\} \backslash\{x\}$. Then $(f h, g) \theta\left(f^{\prime} h, g^{\prime}\right)$ where $|f h|=\left|f^{\prime} h\right|=i-1$ and $\operatorname{dom} f h \neq \operatorname{dom} f h^{\prime}$. Once again applying Theorem 3.9, we conclude that $\theta$ must contain all pairs satisfying conditions (1) or (3).

By symmetrically applying the above considerations to the second argument, we also show that $\theta$ contains the pairs that satisfy condition (2).

The next step is to prove that, for any $Q \in\{\mathcal{T}, \mathcal{P} \mathcal{T}, \mathcal{I}\}$ the pairs that satisfy condition (4) are also in $\theta$.

Note that the group of units of $Q_{m} \times Q_{n}$ is $S_{m} \times S_{n}$. We can choose $(u, v) \in S_{m} \times S_{n}$ such that both $u f$ and $v g$ are idempotent transformations. Now $(u f, v g)=(u, v)(f, g) \theta(u, v)\left(f^{\prime}, g^{\prime}\right)=$ $\left(u f^{\prime}, v g^{\prime}\right)$, and clearly $(u f) \cdot \sigma_{1}=u f^{\prime}$ and $(v g) \cdot \sigma_{2}=v g^{\prime}$. Hence we may assume w.l.o.g. that $f, g$ are idempotents.

Let $H$ be the $\mathcal{H}$-class of $(f, g)$. Then $\left(f^{\prime}, g^{\prime}\right) \in H$. As $H$ contains an idempotent, $H$ is a group. Moreover, it is easy to see that $\phi$ given by $\left(\omega_{1}, \omega_{2}\right) \phi=\left(f \cdot \omega_{1}, g \cdot \omega_{2}\right)$ is an isomorphism from $S_{i} \times S_{k}$ to $H$.

Let $\theta^{\prime}$ be the restriction of $\theta$ to $H$, then $\theta^{\prime}$ is a congruence on a group. Let $K^{\prime}$ be the normal subgroup of $H$ corresponding to $\theta^{\prime}$, and $K^{\prime}=\bar{K} \phi^{-1}$. As an idempotent, $(f, g)$ is the identity of $H$, so $(f, g) \in K^{\prime}$ and hence $\left(f^{\prime}, g^{\prime}\right) \in K^{\prime}$, as $(f, g) \theta^{\prime}\left(f^{\prime}, g^{\prime}\right)$. Applying $\phi^{-1}$, we get that $\left(\sigma_{1}, \sigma_{2}\right) \in K$. As $K$ is a normal subgroup of $S_{i} \times S_{k}$, we obtain $N \subseteq K$. 
Now let us take a pair $((a, b),(c, d))$ that satisfies (4), that is, $|a|=|c|=i,|b|=|d|=k, a \mathcal{H} c$, $b \mathcal{H} d$ such that $a \cdot \tau_{1}=c, b \cdot \tau_{2}=d$ for some $\left(\tau_{1}, \tau_{2}\right) \in N$. It remains to show that $(a, b) \theta(c, d)$.

As $|a|=|f|$ and $|b|=|g|$, we have $a \mathcal{J} f$, and $b \mathcal{J} g$, so there exists $h_{1}, h_{2} \in Q_{m}$ and $h_{3}, h_{4} \in Q_{n}$ such that $a=h_{1} f h_{2}, b=h_{3} g h_{4}$. Once again as $|a|=|f|$ and $|b|=|g|, h_{2} \mid \operatorname{im} f$ is an injection and so is $\left.h_{4}\right|_{\operatorname{im} f}$. Hence w.l.o.g. we may assume that $h_{2} \in S_{m}, h_{4} \in S_{n}$.

Note that as $\left(\tau_{1}, \tau_{2}\right) \in N$, we have $\left(\tau_{1}, \tau_{2}\right) \in K$, since $N \subseteq K$. Recall that $\bar{\tau}_{1}^{\pi_{1}(H)} \in S_{\mathrm{im} \pi_{1}(H)}$ denotes the function such that $h \bar{\tau}_{1}^{\pi_{1}(H)}=h \cdot \tau_{s}$ for all $h \in \pi_{1}(H)$. We will write $\bar{\tau}_{1}$ for the extension of $\bar{\tau}_{s}^{\pi_{1}(H)}$ to $S_{m}$ that is the identity on $\{1, \ldots, m\} \backslash \mathrm{im} H$, and use corresponding notation if $\tau_{1}$ is replaced by other elements of $S_{i}$ or $S_{k}$.

Consider $y=\left(f h_{2} \bar{\tau}_{1} h_{2}^{-1}, g h_{4} \bar{\tau}_{2} h_{4}^{-1}\right)$. It is straightforward to check that $y \in H$, whence $y=\left(f \cdot \omega_{1}, g \cdot \omega_{2}\right)$ for some $\left(\omega_{1}, \omega_{2}\right) \in S_{i} \times S_{k}$. In fact, $\bar{\omega}_{1}=h_{2} \bar{\tau}_{1} h_{2}^{-1}$, as these elements agree on $\operatorname{im} f$ and are the identity otherwise. Hence $\bar{\omega}_{1}$ and $\bar{\tau}_{1}$ are conjugate in $S_{m}$ and so have the same cycle structure. The cycle structure of $\bar{\omega}_{1}$ is obtained from $\omega_{1}$ by the addition of $m-i$ trivial cycles. The same holds for $\bar{\tau}_{1}$ and $\tau_{1}$. It follows that $\tau_{1}$ and $\omega_{1}$ are conjugates in $S_{i}$. Analogously, $\bar{\omega}_{2}=h_{4} \bar{\tau}_{2} h_{4}^{-1}$, and $\tau_{2}$ and $\omega_{2}$ are conjugates in $S_{k}$. Therefore, $\left(\omega_{1}, \omega_{2}\right)$ is a conjugate of $\left(\tau_{1}, \tau_{2}\right)$ in $S_{i} \times S_{k}$, and hence $\left(\omega_{1}, \omega_{2}\right) \in K$.

We obtain that $\left(f \cdot \omega_{1}, f \cdot \omega_{2}\right) \in K^{\prime}$, and hence $(f, g) \theta^{\prime}\left(f \cdot \omega_{1}, f \cdot \omega_{2}\right)$, and so $(f, g) \theta\left(f \cdot \omega_{1}, f \cdot \omega_{2}\right)$. Now $f \cdot \omega_{1}=f \bar{\omega}_{1}, g \cdot \omega_{2}=g \bar{\omega}_{2}$ and so

$$
\begin{aligned}
(a, b)=\left(h_{1} f h_{2}, h_{3} g h_{4}\right) \theta\left(h_{1} f \bar{\omega}_{1} h_{2}, h_{3} g \bar{\omega}_{2} h_{4}\right) & =\left(h_{1} f\left(h_{2} \bar{\tau}_{1} h_{2}^{-1}\right) h_{2}, h_{3} g\left(h_{4} \bar{\tau}_{2} h_{4}^{-1}\right) h_{4}\right) \\
& =\left(h_{1} f h_{2} \bar{\tau}_{1}, h_{3} g h_{4} \bar{\tau}_{2}\right) \\
& =\left(a \bar{\tau}_{1}, b \bar{\tau}_{2}\right)=\left(a \cdot \tau_{1}, b \cdot \tau_{2}\right)=(c, d),
\end{aligned}
$$

as required.

It follows that all pairs that satisfy one of the conditions (1) to (4) are in $\theta$.

Conversely, let $\rho$ be defined on $Q_{m} \times Q_{n}$ by, for all $(a, b),(c, d) \in Q_{m} \times Q_{n}$,

$$
(a, b) \rho(c, d) \text { iff } \begin{cases}(a, b)=(c, d), & \text { or } \\ (a, b) \neq(c, d), & \text { and one of }(1) \text { to }(4) \text { holds. }\end{cases}
$$

We can routinely verify that $\rho$ is a congruence. As $\left((f, g),\left(f^{\prime}, g^{\prime}\right)\right) \in \rho, \theta \subseteq \rho$. We have shown that $\rho \subseteq \theta$, therefore $\theta=\rho$, as required.

We can get a more direct description of the congruence classes by using the following folklore result. Its proof is an easy exercise.

Theorem 3.12. All normal subgroups of $S_{i} \times S_{k}$ are either products of normal subgroups of $S_{i}$ and $S_{k}$ or the group of all pairs $\left(\sigma_{1}, \sigma_{2}\right)$, where $\sigma_{1}$ and $\sigma_{2}$ are permutations with the same signature.

Corollary 3.13. Under the conditions of Theorem 3.11, if one of $\sigma_{1}$ or $\sigma_{2}$ is an even permutation, then $\theta$ agrees on $\left(I_{i} \times I_{j}\right)^{2}$ with the product congruence $\theta_{1} \times \theta_{2}$, and is trivial elsewhere. Concretely, in this case, let $N_{1}, N_{2}$ be the normal subgroups of $S_{i}$ and $S_{k}$ generated by $\sigma_{1}, \sigma_{2}$, respectively. Then for $(a, b) \neq(c, d)$, we have $(a, b) \theta(c, d)$ if and only if one of the following holds:

(1) $|a|,|c| \leq i-1,|b|,|d| \leq k-1$;

(2) $|a|,|c| \leq i-1,|b|=|d|=k, b \mathcal{H} d, d=b \cdot \tau_{2}$ for some $\tau_{2} \in N_{2}$;

(3) $|a|=|c|=i,|b|,|d| \leq k-1, a \mathcal{H} c, a=c \cdot \tau_{1}$ for some $\tau_{1} \in N_{1}$;

(4) $|a|=|c|=i,|b|=|d|=k, a \mathcal{H} c, b \mathcal{H} d, a=c \cdot \tau_{1}$ for some $\tau_{1} \in N_{1}$, and $d=b \cdot \tau_{2}$ for some $\tau_{2} \in N_{2}$.

Under the conditions of Theorem 3.11, if both $\sigma_{1}, \sigma_{2}$ are odd permutations, then for $(a, b) \neq$ $(c, d)$, we have $(a, b) \theta(c, d)$ if and only if one of the following holds:

(1) $|a|,|c| \leq i-1,|b|,|d| \leq k-1$;

(2) $|a|,|c| \leq i-1,|b|=|d|=k, b \mathcal{H} d$;

(3) $|a|=|c|=i,|b|,|d| \leq k-1, a \mathcal{H} c$;

(4) $|a|=|c|=i,|b|=|d|=k, a \mathcal{H} c, b \mathcal{H} d$ and there exist $\tau_{1} \in S_{i}, \tau_{2} \in S_{k}$ of the same signature such that $a \cdot \tau_{1}=c, b \cdot \tau_{2}=d$. 


\section{The structure of All CONGRUences on $Q_{m} \times Q_{n}$}

We now look at our main aim: to determine the structure of all congruences on $Q_{m} \times Q_{n}$. When studying a congruence $\theta$ on $Q_{m} \times Q_{n}$, as in the case of $Q_{n}$ (Theorem 2.2), we realize that the $\theta$-classes are intrinsically related to the $\mathcal{D}$-classes of $Q_{m} \times Q_{n}$. We shall show that $\theta$ is determined by some minimal blocks of $\theta$-classes, called here $\theta$-dlocks, which are also unions of $\mathcal{D}$-classes. The strategy will be to determine the possible types of $\theta$-dlocks and to describe $\theta$ within such blocks.

Throughout this section, $\theta$ denotes a congruence on $Q_{m} \times Q_{n}$. To avoid some minor technicalities, we assume that $Q_{m}$ and $Q_{n}$ are non-trivial, i.e. we exclude the factors $\mathcal{T}_{0}, \mathcal{T}_{1}, \mathcal{P} \mathcal{T}_{0}$, $\mathcal{I}_{0}$.

Definition 4.1. A $\theta$-dlock $X$ is a non-empty subset of $Q_{m} \times Q_{n}$ such that

(1) $X$ is a union of $\theta$-classes as well as a union of $\mathcal{D}$-classes;

(2) No proper non-empty subset of $X$ satisfies (1).

In other words, the $\theta$-dlocks are the classes of the equivalence relation generated by $\mathcal{D} \cup \theta$. We will just write dlock if $\theta$ is understood by context, and will describe dlocks by listing their $\mathcal{D}$-classes. Concretely, let $D_{i, j}$ be the $\mathcal{D}$-class of all pairs $(f, g)$ such that $|f|=i,|g|=j$. Recall that $0 *$ refers to 0 for $Q \in\{\mathcal{I}, \mathcal{P} \mathcal{T}\}$ and to 1 for $Q=\mathcal{T}$. For $P \subseteq\{0 *, \ldots, m\} \times\{0 *, \ldots, n\}$, we set $D_{P}=\cup_{(i, j) \in P} D_{i, j}$.

First we describe the various configurations of dlocks with respect to the $\mathcal{H}$-classes they contain. To this end, we will divide the dlocks into 9 different types.

Definition 4.2. Let $X$ be a $\theta$-dlock. We say that $X$ has first component type

$\varepsilon:$ if for all $(a, b),(c, d) \in X$ with $(a, b) \theta(c, d)$ we have $a=c$;

$\mathcal{H}$ : if there exist $(a, b),(c, d) \in X$ such that $a \neq c$ and $(a, b) \theta(c, d)$, and for all $\left(a^{\prime}, b^{\prime}\right)$, $\left(c^{\prime}, d^{\prime}\right) \in X$ such that $\left(a^{\prime}, b^{\prime}\right) \theta\left(c^{\prime}, d^{\prime}\right)$ we have $a^{\prime} \mathcal{H} c^{\prime}$;

$F$ : if there exist $(a, b),(c, d) \in X$ such that $(a, c) \notin \mathcal{H}$ and $(a, b) \theta(c, d)$.

We define the second component type of $X$ dually. Finally we say that $X$ has type $V W$ for $V, W \in\{\varepsilon, \mathcal{H}, F\}$ if it has first component type $V$ and second component type $W$.

Clearly, we obtain all possible types of dlocks. Next, we will describe the congruence $\theta$ by means of its restriction to each of its dlocks.

Lemma 4.3. Let $X=D_{P}=\cup_{(i, j) \in P} D_{i, j}$ be a $\theta$-dlock of type $F F$. Then $P$ is a downwardclosed subset of $(\{0 *, \ldots, m\} \times\{0 *, \ldots, n\}, \leq \times \leq)$, and $X$ is a single $\theta$-class that is an ideal of $Q_{m} \times Q_{n}$. Conversely, let $\theta$ have an ideal class $I$, and let $P \subseteq\{0 *, \ldots, m\} \times\{0 *, \ldots, n\}$ be the set of pairs $(i, j)$ for which $D_{i, j} \cap I \neq \emptyset$. Then $I$ is a dlock of Type $F F$ unless $Q \in\{\mathcal{I}, \mathcal{P} \mathcal{T}\}$ and one of the following holds:

(1) $P=\{0\} \times\{0, \ldots, j\}$ for some $j \in\{0, \ldots, n\}$;

(2) $P=\{0, \ldots, i\} \times\{0\}$ for some $i \in\{0, \ldots, m\}$.

Proof. Let $X$ be a $\theta$-dlock of type $F F$ and assume that its first component type $F$ is witnessed by $\left((f, g),\left(f^{\prime}, g^{\prime}\right)\right)$, i.e. $\left((f, g),\left(f^{\prime}, g^{\prime}\right)\right) \in \theta \cap X^{2}$ and $\left(f, f^{\prime}\right) \notin \mathcal{H}$.

Let $\theta^{\prime}$ be the congruence generated by $\left((f, g),\left(f^{\prime}, g^{\prime}\right)\right)$. Let $i=\max \left\{|f|,\left|f^{\prime}\right|\right\}$ and $k=$ $\max \left\{|g|,\left|g^{\prime}\right|\right\}$. Consider the ideals $I_{i} \subseteq Q_{m}, I_{k} \subseteq Q_{n}$. By either Theorem 3.9 (if $g \mathcal{H} g^{\prime}, g \neq g$ ), Theorem 3.7 (if $\left(g, g^{\prime}\right) \notin \mathcal{H}$ ) or Lemma 3.4 (if $g=g^{\prime}$ ), all sets of the form $I_{i} \times\{b\}$, where $|b| \leq k$, are contained in congruence classes of $\theta^{\prime}$. By a dual argument $\{a\} \times I_{k}$ is contained in a $\theta^{\prime}$ class for $|a| \leq i$. By choosing $|a|=0 *=|b|$, we see that these sets intersect. It follows that $\theta^{\prime}$ and hence $\theta$ have a class that contains $D_{0 *, 0 *}$. It is straightforward to check that such a $\theta$-congruence class $Y$ is an ideal of $Q_{m} \times Q_{n}$. By Lemma 3.2, all ideals are unions of $\mathcal{D}$-classes. Therefore $Y$ is a dlock that contains $X$. As dlocks are disjoint $X=Y$, and so $X$ is a single $\theta$-class that is an ideal.

Conversely, suppose that $I$ is an ideal class of $\theta$, and that $P$ indexes the $\mathcal{D}$-classes intersecting $I$. By Lemma 3.2, the ideal $I$ is a union of $\mathcal{D}$-classes, so that $D_{P}=I$, and $I$ is a dlock. It is a 
dlock of type $F F$ unless either $\pi_{1}(I)$ or $\pi_{2}(I)$ consists of a single $\mathcal{H}$-class. The listed exceptions are the only way this can happen, as we assumed that $Q_{n}, Q_{m}$ are non-trivial.

In particular, by Lemma 3.3, the congruence $\theta$ has at most one dlock of type $F F$. If it exists, the unique dlock of type $F F$ is the $\theta$-class that contains $D_{0 *, 0 *}$. By Lemma 3.2, we can visualize this dlock as a "landscape" (see Figure 1).

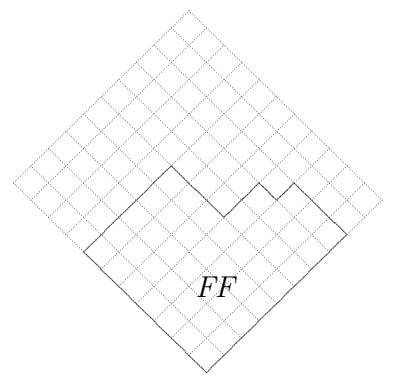

Figure 1. A possible configuration for a dlock of type $F F$

Lemma 4.4. Let $X$ be a $\theta$-dlock. Then $X$ is of type $\varepsilon F$ or $\mathcal{H} F$ if and only if there exist $0 * \leq i \leq m, 1 \leq j \leq n$, and $N \unlhd S_{i}$, such that $X=D_{P}$ with $P=\{i\} \times\{0 *, \ldots, j\}$, and for every $(f, g) \in X$,

$$
[(f, g)]_{\theta}=\left\{\left(f^{\prime}, g^{\prime}\right) \in X \mid f \mathcal{H} f^{\prime} \text { and } f^{\prime}=f \cdot \sigma \text { for some } \sigma \in N\right\} .
$$

If $X$ satisfies these requirements, then $X$ is of type $\mathcal{H} F$ exactly when $N \neq \varepsilon_{i}$.

Proof. Let $X=D_{P}$ be a $\theta$-dlock. Suppose $X$ is of type $\varepsilon F$ or $\mathcal{H} F$. Then for every $\theta$-class $C$ contained in $X$, we have $\pi_{1}(C)$ contained in an $\mathcal{H}$-class of $Q_{m}$ by the definitions of first component types $\varepsilon$ and $\mathcal{H}$. Hence $\pi_{1}(C)$ is contained in a $\mathcal{D}$-class of $Q_{m}$ for all such $C$. Since the elements of $\pi_{1}(X)$ that are $\theta$-related are pairwise either equal or $\mathcal{H}$-related, $\pi_{1}(X)$ must be a single $\mathcal{D}$-class of $Q_{m}$, say $D_{i}$, by the definition of $\theta$-dlock. Therefore $P=\{i\} \times K$ for some non-empty $K \subseteq\{0 *, \ldots, n\}$. Let $j$ be the largest element in $K$. We claim that there exists $\left((f, g),\left(f^{\prime}, g^{\prime}\right)\right) \in \theta$ with $\left(g, g^{\prime}\right) \notin \mathcal{H}$ and $(f, g) \in D_{i, j}$. For otherwise $[(\bar{f}, \bar{g})]_{\theta}$ would be contained in an $\mathcal{H}$-class for every $(\bar{f}, \bar{g}) \in D_{i, j}$, and then $\{(i, j)\}$ would index a dlock contained in $X$, and $X$ being minimal would imply $D_{i, j}=X$. However, $X$ is a dlock of type $\epsilon F$ or $\mathcal{H} F$, and we have a contradiction.

So there exists $\left((f, g),\left(f^{\prime}, g^{\prime}\right)\right) \in \theta$, such that $\left(g, g^{\prime}\right) \notin \mathcal{H}$ and $(f, g) \in D_{i, j}$. Now, there exists $\sigma \in S_{i}$ such that $f^{\prime}=f \cdot \sigma$. Let $N_{\sigma}$ be the normal subgroup of $S_{i}$ generated by $\sigma$. Let $N$ be maximal among all such $N_{\sigma}$. Assume that $\left((f, g),\left(f^{\prime}, g^{\prime}\right)\right)$ witnesses $N$.

Let $\theta^{\prime}$ be the principal congruence generated by $\left((f, g),\left(f^{\prime}, g^{\prime}\right)\right)$. Either Corollary 3.5 or the dual of Theorem 3.9 is applicable to $\theta^{\prime}$ - the first one if $f=f^{\prime}$, and hence $N=\varepsilon_{i}$, and the second one otherwise.

Assume first that $N \neq \varepsilon_{i}$, then by the dual of Theorem 3.9, we get that for all $(\hat{f}, \hat{g}) \in$ $D_{\{i\} \times\{0 *, \ldots, j\}}$,

$$
[(\hat{f}, \hat{g})]_{\theta^{\prime}}=\{(\bar{f}, \bar{g})|| \bar{g} \mid \leq j, \bar{f}=\hat{f} \cdot \bar{\sigma} \text { for some } \bar{\sigma} \in N\} .
$$

If $N=\varepsilon_{i}$ then by Corollary 3.5, we get that for all $(\hat{f}, \hat{g}) \in D_{\{i\} \times\{0 *, \ldots, j\}}$,

$$
[(\hat{f}, \hat{g})]_{\theta^{\prime}}=\{(\hat{f}, \bar{g})|| \bar{g} \mid \leq j\}=\{(\bar{f}, \bar{g})|| \bar{g} \mid \leq j, \bar{f}=\hat{f} \cdot \bar{\sigma} \text { for some } \bar{\sigma} \in N\} .
$$

As the rightmost expressions in (2) and (3) are identical, we may treat both cases simultaneously.

We claim that the sets from (2) or (3) are also congruence classes of $\theta$. As $\theta^{\prime} \subseteq \theta$ the sets in (2), (3) are contained in classes of $\theta$. We have already established that $P \subseteq\{i\} \times\{0 *, \ldots, j\}$, it now follows that $P=\{i\} \times\{0 *, \ldots, j\}$, since the sets in (2), (3) intersect all $\mathcal{D}$-classes indexed by $\{i\} \times\{0 *, \ldots, j\}$.

Now let $E$ be a congruence class of $\theta$ that is contained in $X$. Then $E$ must be a union of sets from (2) or (3), and in particular, must intersect $D_{i, j}$, say $(\hat{f}, \hat{g}) \in E \cap D_{i, j}$. Let $(\bar{f}, \bar{g}) \theta(\hat{f}, \hat{g})$. Then $|\bar{g}| \leq j$, since $E \subseteq X=D_{P}$. Moreover $(\hat{f}, \bar{f}) \in \mathcal{H}$ as $X$ is a dlock of type $\varepsilon F$ or $\mathcal{H} F$. Now, 
if $\beta$ is given by $\bar{f}=\hat{f} \cdot \beta$, then $\beta \in N$, by the maximality of $N$. It follows that $E$ is one of the sets from $(2),(3)$, and thus contained in $[(\hat{f}, \hat{g})]_{\theta^{\prime}}$. Therefore $[(\hat{f}, \hat{g})]_{\theta}=[(\hat{f}, \hat{g})]_{\theta^{\prime}}$, for $(\hat{f}, \hat{g}) \in X$.

Notice that $j$ cannot be 0 . In fact, if $j=0$, then $\pi_{2}(X)$ only contains one element, which contradicts the definition of type $\epsilon F$ or $\mathcal{H} F$. We have concluded the proof of the "if" direction of the first statement of the lemma.

The "only if" direction now follows directly from the description (1), provided that there exists $\left(g, g^{\prime}\right) \notin \mathcal{H},\left(g, g^{\prime}\right) \in \pi_{2}(X)$. This holds as $Q_{n}$ is non-trivial and $j \geq 1$.

Finally, the last statement follows directly from (1) and the definitions of type $\varepsilon F$ or $\mathcal{H} F$.

If $X$ is a dlock of type $\mathcal{H} F$ or $\varepsilon F$, we call the group $N \unlhd S_{i}$ from Lemma 4.4 the normal subgroup associated with $X$. Clearly, a dual version of Lemma 4.4 holds for dlocks $X$ of type $F \varepsilon$ or $F \mathcal{H}$.

Lemma 4.5. Let $X=D_{P}$ be a dlock of type $\mathcal{H} F$ with $P=\{i\} \times\{0 *, \ldots, j\}$ and associated normal subgroup $N \unlhd S_{i}$. Then $i \geq 2$, and $\{0 *, \ldots, i-1\} \times\{0 *, \ldots, j\}$ is contained in the index set of a $\theta$-dlock of type $F F$.

Moreover, every congruence $\theta$ has at most one dlock of type $\mathcal{H} F$.

Proof. By Lemma 4.4, we have $\varepsilon_{i} \neq N \unlhd S_{i}$, which implies that $i \geq 2$. By the description (1), we may find $\left((f, g),\left(f^{\prime}, g^{\prime}\right)\right) \in \theta \cap X^{2}$, such that $(f, g) \in D_{i, j}, f \neq f^{\prime},\left(f, f^{\prime}\right) \in \mathcal{H}$, and $\left(g, g^{\prime}\right) \notin \mathcal{H}$. As $X=D_{P},\left|g^{\prime}\right| \leq j,|f|=i$.

Let $\theta^{\prime}$ be the principal congruence generated by $\left((f, g),\left(f^{\prime}, g^{\prime}\right)\right)$. Then $\theta^{\prime}$ is described in the dual of Corollary 3.10. By this corollary, if $C:=\{0 *, \ldots, i-1\} \times\{0 *, \ldots, j\}$ then $D_{C}$ is one equivalence class of $\theta^{\prime}$, and hence contained in an equivalence class of $\theta$. As $j \geq 1, i-1 \geq 1$, and we excluded the case that $Q_{n}$ is trivial, $\pi_{1}\left(D_{C}\right)$ and $\pi_{2}\left(D_{C}\right)$ both contain more then one $\mathcal{H}$-class. Hence $C$ is contained in the index set of a $\theta$-dlock $\bar{X}$ of type $F F$.

Now assume that $\theta$ has a potentially different dlock $X^{\prime}=D_{P^{\prime}}$ of type $\mathcal{H} F$, where $P^{\prime}=$ $\left\{i^{\prime}\right\} \times\left\{0 *, \ldots, j^{\prime}\right\}$. By applying our previous results to $X^{\prime}$, we get that $D_{i^{\prime}-1,0 *}$ must also lie in a $\theta$-dlock $\bar{X}$ of type $F F$. As noted after Lemma 4.3, dlocks of type $F F$ are unique, and so $D_{i^{\prime}-1,0 *} \subseteq \bar{X}$. So both $D_{i-1,0 *} \subseteq \bar{X}$ and $D_{i^{\prime}-1,0 *} \subseteq \bar{X}$, but $D_{i, 0 *} \nsubseteq \bar{X}$ and $D_{i^{\prime}, 0 *} \nsubseteq \bar{X}$, as these $\mathcal{D}$-classes lie in the $\mathcal{H} F$-dlocks $X$ and $X^{\prime}$. By Lemma 4.3, the index set of $\bar{X}$ is downwards closed, hence there is a unique $\bar{i}$ such that $D_{\bar{i}, 0 *} \subseteq \bar{X}, D_{\bar{i}+1,0 *} \nsubseteq \mathbb{X}$. It follows that $i=\bar{i}+1=i^{\prime}$. Thus both $X$ and $X^{\prime}$ contain $D_{i, 0 *}$ and therefore $X=X^{\prime}$.

We may visualize the statement of Lemma 4.5 by saying that a $\theta$-dlock of type $\mathcal{H} F$ must lie on the "most eastern slope" of the dlock of type $F F$. Once again, a dual result holds for dlocks of type $F \mathcal{H}$. Figure 2 shows the possible positions for dlocks of type $\mathcal{H} F$ and $F \mathcal{H}$ in relation to a dlock of type $F F$.

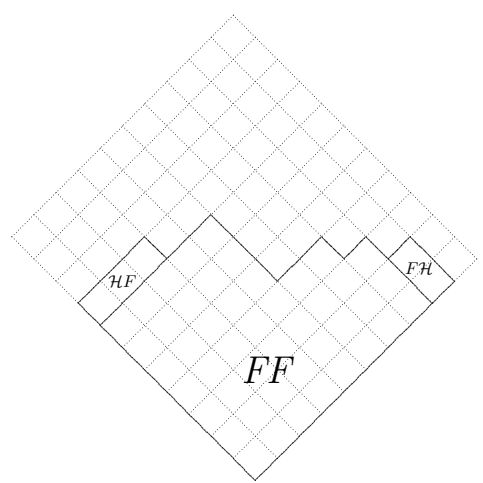

Figure 2. A possible configuration for three dlocks of type $F F, \mathcal{H F}$ and $F \mathcal{H}$

Lemma 4.6. Let $X=D_{P}$ be a $\theta$-dlock of type $\varepsilon F$ with $P=\{i\} \times\{0 *, \ldots, j\}$. Then for each $k=0 *, \ldots, i-1$, the set $\{k\} \times\{0 *, \ldots, j\}$ is contained in the index set of a $\theta$-dlock of type $F F$, $\mathcal{H} F$, or $\varepsilon F$.

Proof. If $i=0 *$, the statement quantifies over the empty set, so assume this is not the case. It suffices to show the statement for the case that $k=i-1$, the remaining values follow by 
induction applying: the same result if the resulting $\theta$-dlock $\bar{X}$ is of type $\varepsilon F$; Lemma 4.3 if $\bar{X}$ is of type $F F$; or Lemma 4.5 if $\bar{X}$ is of type $\mathcal{H F}$.

By Lemma 4.4 we may find $\left((f, g),\left(f, g^{\prime}\right)\right) \in \theta \cap X^{2}$, such that $(f, g) \in D_{i, j},\left(g, g^{\prime}\right) \notin \mathcal{H}$. Note that this implies that $\left|g^{\prime}\right| \leq j$.

Let $\theta^{\prime}$ be the principal congruence generated by $\left((f, g),\left(f, g^{\prime}\right)\right)$. Then $\theta^{\prime}$ is described in the dual of Corollary 3.10. By this corollary, if $|\bar{f}|=i-1$, then

$$
[(\bar{f}, g)]_{\theta^{\prime}}=\{(\bar{f}, \hat{g})|| \hat{g} \mid \leq j\} .
$$

It follows that $\{i-1\} \times\{0 *, \ldots, j\}$ is the index set of a $\theta^{\prime}$-dlock and hence contained in the index set of a $\theta$-dlock $X^{\prime}$. As $\left((\bar{f}, g),\left(\bar{f}, g^{\prime}\right)\right) \in \theta^{\prime} \subseteq \theta$, and $\left(g, g^{\prime}\right) \notin \mathcal{H}$, it follows that $X^{\prime}$ is of type $F F, \mathcal{H F}$, or $\varepsilon F$, as these three options cover all cases where $\pi_{2}\left(D_{P^{\prime}}\right) \nsubseteq \mathcal{H}$. The result follows.

Lemma 4.7. For a given congruence $\theta$, let $J$ be the set of values $\left(i, j_{i}\right)$ such that $\{i\} \times\left\{0 *, \ldots, j_{i}\right\}$ is the index set of a $\theta$-dlock of type $\varepsilon F$. Then $\pi_{1}(J)$ is a set of consecutive integers (possibly empty), and the values $j_{i}$ are non-increasing in $i$.

Proof. Assume that $i_{1}<i_{2}<i_{3}$ with $i_{1}, i_{3} \in \pi_{1}(J)$. We want to show that $i_{2} \in \pi_{1}(J)$, as well. As $i_{1}, i_{3} \in \pi_{1}(J), X_{1}=D_{\left\{i_{1}\right\} \times\left\{0 *, \ldots, j_{i_{1}}\right\}}$ and $X_{3}=D_{\left\{i_{3}\right\} \times\left\{0 *, \ldots, j_{i_{3}}\right\}}$ are $\theta$-dlocks of type $\varepsilon F$, for some $j_{i_{1}}, j_{i_{3}}$. Applying Lemma 4.6 to $X_{3}$, we get that $D_{\left\{i_{2}\right\} \times\left\{0 *, \ldots, j_{i_{3}}\right\}}$ is contained in $\theta$-dlock $X^{\prime}$ of type $F F, \mathcal{H} F$, or $\varepsilon F$.

If $X^{\prime}$ were of type $\mathcal{H} F$ or $F F$, then by Lemma 4.5 or Lemma 4.3 , respectively, $D_{\left\{i_{1}\right\} \times\left\{0 *, \ldots, j_{3}\right\}}$ would be contained in a dlock of type $F F$, which in the latter case would be the dlock $X^{\prime}$ itself. In particular, $D_{i_{1}, 0 *}$ would be contained in a dlock of type $F F$. However, as $i_{1} \in J, D_{i_{1}, 0 *}$ is contained in the dlock $X_{1}$ of type $\varepsilon F$, a contradiction. Hence $X^{\prime}$ is of type $\varepsilon F$, and $i_{2} \in \pi_{1}(J)$. It follows that $\pi_{1}(J)$, if not empty, is a set of consecutive integers.

Now let $\left(i, j_{i}\right),\left(i-1, j_{i-1}\right) \in J$. As above, we have that $D_{\{i-1\} \times\left\{0 *, \ldots, j_{i}\right\}}$ is contained in a $\theta$-dlock $X^{\prime}$ of type $F F, \mathcal{H} F$, or $\varepsilon F$. As $\left(i-1, j_{i-1}\right) \in J$, this must necessarily be in the dlock $D_{\{i-1\} \times\left\{0 *, \ldots, j_{i-1}\right\}}$ of type $\varepsilon F$. Hence $j_{i} \leq j_{i-1}$, and so the values $j_{i}$ are non-increasing in $i$.

Lemma 4.6 and Lemma 4.7 show that, if there are any $\theta$-dlocks of type $\varepsilon F$, they are layered on the top of each other without "overhanging", with the lowest one either starting at $i=0 *$, or lying on the top of the eastern most slope of the dlock of type $F F$, or lying on the top of the dlock of type $\mathcal{H F}$, in the last two cases without overhanging the dlocks below them. An example is depicted in Figure 3. Once again, the dual versions of Lemmas 4.6 and 4.7 hold as well.

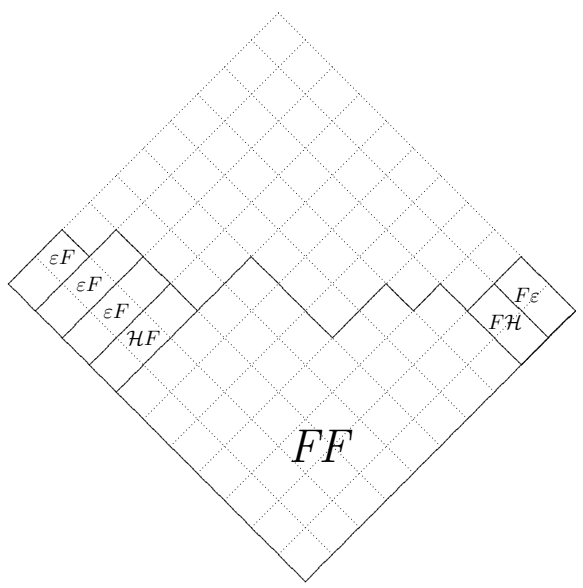

Figure 3. A possible configuration for dlocks of type $F F, \mathcal{H F}, F \mathcal{H}, \varepsilon F$, and $F \varepsilon$ 
Lemma 4.8. Let $X$ be a dlock. Then $X$ is of type $\mathcal{H} \mathcal{H}, \varepsilon \mathcal{H}, \mathcal{H} \varepsilon$, or $\varepsilon \varepsilon$ if and only if $X=D_{i, j}$ for some $i, j$ and there exists $N \unlhd S_{i} \times S_{j}$ such that for every $(f, g) \in X$,

$$
\begin{aligned}
{[(f, g)]_{\theta}=} & \left\{\left(f^{\prime}, g^{\prime}\right) \in D_{i, j} \mid f \mathcal{H} f^{\prime}, g \mathcal{H} g^{\prime},\right. \\
& \text { and } \left.\left(f^{\prime}, g^{\prime}\right)=(f \cdot \sigma, g \cdot \tau) \text { for some }(\sigma, \tau) \in N\right\} .
\end{aligned}
$$

Moreover, in this situation,

(a) $X$ is of type $\mathcal{H} \mathcal{H} \Longleftrightarrow \pi_{1}(N) \neq \varepsilon_{i}$ and $\pi_{2}(N) \neq \varepsilon_{j}$;

(b) $X$ is of type $\varepsilon \mathcal{H} \Longleftrightarrow N=\varepsilon_{i} \times N^{\prime}$ for some $N^{\prime} \neq \varepsilon_{j}$;

(c) $X$ is of type $\mathcal{H} \varepsilon \Longleftrightarrow N=N^{\prime} \times \varepsilon_{j}$ for some $N^{\prime} \neq \varepsilon_{i}$;

(d) $X$ is of type $\varepsilon \varepsilon \Longleftrightarrow N=\varepsilon_{i} \times \varepsilon_{j}$.

Proof. Let $X$ be of type $\mathcal{H} \mathcal{H}, \varepsilon \mathcal{H}, \mathcal{H} \varepsilon$, or $\varepsilon \varepsilon$. Then $\pi_{1}\left(\theta \cap X^{2}\right) \subseteq \mathcal{H}$ and $\pi_{2}\left(\theta \cap X^{2}\right) \subseteq \mathcal{H}$. It follows that each $\mathcal{H}$-class in $X$ is a union of $\theta$-classes. Therefore every $\mathcal{D}$-class in $X$ is a union of $\theta$-classes as well, and by the minimality property of a dlock, there is only one $\mathcal{D}$-class in $X$. It follows that $X=D_{i, j}$ for some $(i, j)$.

Now as $\theta \cap X^{2} \subseteq \mathcal{H} \times \mathcal{H}$, having $\left((f, g),\left(f^{\prime}, g^{\prime}\right)\right) \in \theta \cap X^{2}$ implies that $f^{\prime}=f \cdot \sigma, g^{\prime}=g \cdot \tau$ for some $\sigma \in S_{i}$ and $\tau \in S_{j}$. Let $N \subseteq S_{i} \times S_{j}$ be the set of all $(\sigma, \tau)$ that correspond to some $\left((f, g),\left(f^{\prime}, g^{\prime}\right)\right) \in \theta \cap X^{2}$.

If $N=\left\{\left(\operatorname{id}_{S_{i}}, \operatorname{id}_{S_{j}}\right)\right\}$ then $\theta$ is the identity on $X$, and $\theta \cap X^{2}$ is given by (4) with the choice $N=\varepsilon_{i} \times \varepsilon_{j}$.

Otherwise, let $\left(\operatorname{id}_{S_{i}}, \operatorname{id}_{S_{j}}\right) \neq(\sigma, \tau) \in N$, as witnessed by $((f, g),(f \cdot \sigma, g \cdot \tau)) \in \theta \cap X^{2}$. Let $\theta^{\prime}$ be the principal congruence generated by $((f, g),(f \cdot \sigma, g \cdot \tau))$, and let $(\bar{f}, \bar{g}) \in X$. By either Theorem 3.11 (if $\sigma \neq \mathrm{id}_{S_{i}}$ and $\tau \neq \operatorname{id}_{S_{j}}$ ) or Lemma 3.4 and its dual (otherwise), $((\bar{f}, \bar{g}),(\bar{f} \cdot \sigma, \bar{g} \cdot g)) \in \theta^{\prime} \subseteq \theta$. By repeated use of this argument, we get that the equivalence relation given by (4) is contained in $\theta$. As the reverse inclusion follows from the definition of $N$, the expression (4) describes $\theta \cap X^{2}$

It remains to show that $N$ is a normal subgroup of $S_{i} \times S_{j}$. Let $(\sigma, \tau),\left(\sigma^{\prime}, \tau^{\prime}\right) \in N$, and $(f, g) \in X$ arbitrary, then

$$
\left.(f \cdot \sigma, g \cdot \tau) \in \theta \Rightarrow\left(\left((f \cdot \sigma) \cdot \sigma^{\prime}\right),\left((g \cdot \tau) \cdot \tau^{\prime}\right)\right)\right) \in \theta \Rightarrow\left(\left(f \cdot\left(\sigma \sigma^{\prime}\right)\right),\left(g \cdot\left(\tau \tau^{\prime}\right)\right) \in \theta,\right.
$$

where the first implication follows as (4) describes $\theta$ on $X$, and the second implication as - is a group action. Thus $N$ is a subgroup of $S_{i} \times S_{j}$. Now if $(\sigma, \tau) \in N$, then by either Theorem 3.11, Lemma 3.4, or the dual of Lemma 3.4 (applied to any $\left.((f, g),(f \cdot \sigma, g \cdot \tau)) \in \theta \cap X^{2}\right), N$ contains the normal subgroup generated by $(\sigma, \tau)$. Therefore $N$ is a subgroup generated by a union of normal subgroups, and hence it is itself normal.

The converse statement is immediate, and the characterization of the various types follows directly from the definition of the types and from (4).

If $X=D_{i, j}$ is a dlock of type $\mathcal{H} \mathcal{H}, \varepsilon \mathcal{H}, \mathcal{H} \varepsilon$, or $\varepsilon \varepsilon$, we will call $N \unlhd S_{i} \times S_{j}$ from Lemma 4.8 the normal subgroup associated with $X$.

Lemma 4.9. Let $X=D_{i, j}$ be a $\theta$-dlock of type $\mathcal{H} \mathcal{H}$ with normal subgroup $N \unlhd S_{i} \times S_{j}$. Then $i, j \geq 2$, and $D_{i, j-1}$ is contained in either a $\theta$-dlock of type $F F$, or in a $\theta$-dlock of type $\mathcal{H} F$ with normal subgroup $N^{\prime} \unlhd S_{i}$, where $\pi_{1}(N) \subseteq N^{\prime}$.

Symmetrically, $D_{i-1, j}$ is contained in either a $\theta$-dlock of type $F F$, or in a $\theta$-dlock of type $F \mathcal{H}$ with normal subgroup $N^{\prime} \unlhd S_{j}$, where $\pi_{2}(N) \subseteq N^{\prime}$.

Proof. By Lemma 4.8, we have $\pi_{1}(N) \neq \varepsilon_{i}, \pi_{2}(N) \neq \varepsilon_{j}$, and so $i, j \geq 2$. Also by Lemma 4.8, there are $\left((f, g),\left(f^{\prime}, g^{\prime}\right)\right) \in \theta \cap X^{2}$ with $f \neq f^{\prime}, g \neq g^{\prime}$. Let us fix such a pair, and let $\theta^{\prime}$ be the principal congruence generated by it. Then $\theta^{\prime} \subseteq \theta$ and $\theta^{\prime}$ is described in Theorem 3.11.

Let $\bar{g}, \bar{g}^{\prime} \in Q_{m}$ be transformations of rank $j-1$ that are in different $\mathcal{H}$-classes. Such elements clearly exist. By Theorem 3.11(2), we get $\left((f, \bar{g}),\left(f^{\prime}, \bar{g}^{\prime}\right)\right) \in \theta^{\prime} \subseteq \theta$. As $f \neq f^{\prime},\left(\bar{g}, \bar{g}^{\prime}\right) \notin \mathcal{H}$, the $\theta$-dlock $X^{\prime}$ containing $D_{i, j-1}$ is either of type $F F$ or $\mathcal{H} F$, depending on the existence or not of a pair $\left((\hat{f}, \hat{g}),\left(\hat{f}^{\prime}, \hat{g}^{\prime}\right)\right) \in \theta \cap D_{i, j}$ with $\left(\hat{f}, \hat{f}^{\prime}\right) \notin \mathcal{H}$. 
In the first case, we are done, so assume that $X^{\prime}$ is of type $\mathcal{H} F$. Then the restriction of $\theta$ to $X^{\prime 2}$ is given in Lemma 4.4. Let $N^{\prime} \unlhd S_{i}$ be the normal subgroup of $X^{\prime}$. We now wish to prove that $\pi_{1}(N) \subseteq N^{\prime}$.

Let $\sigma \in \pi_{1}(N)$, so $(\sigma, \tau) \in N$ for some $\tau$. Then $((f, g),(f \cdot \sigma, g \cdot \tau)) \in \theta$ by Lemma 4.8, and so $((f, g \bar{g}),(f \cdot \sigma,(g \cdot \tau) \bar{g})) \in \theta$. As $(f, g \bar{g})$ and $(f \cdot \sigma,(g \cdot \tau) \bar{g})$ lie in the $\mathcal{H} F$-dlock $X^{\prime}$, we get that $\pi_{1}(\sigma, \tau)=\sigma \in N^{\prime}$ by Lemma 4.4.

The last statement follows dually.

The result means that the dlocks of type $\mathcal{H} \mathcal{H}$ can only occupy the "valleys" in the landscape formed by the dlocks of $F F, \mathcal{H F}$, and $F \mathcal{H}$ (see Figure 4).

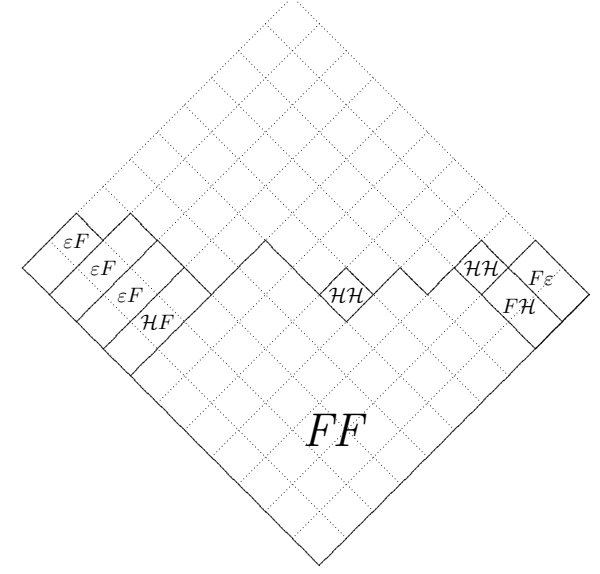

Figure 4. A possible configuration for dlocks of type $F F, \mathcal{H F}, F \mathcal{H}, \varepsilon F, F \varepsilon$, and $\mathcal{H} \mathcal{H}$

Lemma 4.10. Let $X=D_{i, j}$ be a $\theta$-dlock of type $\varepsilon \mathcal{H}$ with normal subgroup $N=\varepsilon_{i} \times N^{\prime} \unlhd S_{i} \times S_{j}$. Then $j \geq 2$, and $D_{i, j-1}$ is contained in a dlock of type $F F, \mathcal{H} F$, or $\varepsilon F$.

Moreover, if $i>0 *$, then $D_{i-1, j}$ is contained in one of the following:

(1) a dlock of type FF;

(2) a dlock of type $\mathcal{H} F$;

(3) a dlock of type $\varepsilon F$;

(4) a dlock of type $F \mathcal{H}$ with normal subgroup $\bar{N} \unlhd S_{j}$ such that $N^{\prime} \subseteq \bar{N}$;

(5) a dlock of type $\mathcal{H} \mathcal{H}$ with normal subgroup $\bar{N} \unlhd S_{i-1} \times S_{j}$ such that $\varepsilon_{i-1} \times N^{\prime} \subseteq \bar{N}$;

(6) a dlock of type $\varepsilon \mathcal{H}$ with normal subgroup $\bar{N} \unlhd S_{i-1} \times S_{j}$ such that $\varepsilon_{i-1} \times N^{\prime} \subseteq \bar{N}$.

Proof. As $N^{\prime}$ is non-trivial, $j \geq 2$. Let $\sigma$ generate $N^{\prime}$ as a normal subgroup in $S_{j}$. Let $(f, g) \in X$ and set $g^{\prime}=g \cdot \sigma$. Then $\left((f, g),\left(f, g^{\prime}\right)\right) \in \theta$. Let $\theta^{\prime}$ be the principal congruence generated by this pair. Then $\theta^{\prime} \subseteq \theta$ and $\theta^{\prime}$ is described in Lemma 3.4.

Let $\bar{g}, \bar{g}^{\prime} \in Q_{m}$ both be transformations of rank $j-1$ that are in different $\mathcal{H}$-classes. Such elements clearly exist. By Lemma 3.4, $\left((f, \bar{g}),\left(f, \bar{g}^{\prime}\right)\right) \in \theta^{\prime} \subseteq \theta$. As $\left(\bar{g}, \bar{g}^{\prime}\right) \notin \mathcal{H}$, the $\theta$-dlock $X^{\prime}$ containing $D_{i, j-1}$ is either of type $F F, \mathcal{H} F$, or $\varepsilon F$, depending on if there exists $\left((\hat{f}, \hat{g}),\left(\hat{f}^{\prime}, \hat{g}^{\prime}\right)\right) \in$ $\theta \cap X^{2}$ with $\left(\hat{f}, \hat{f}^{\prime}\right) \notin \mathcal{H}$ or $\hat{f} \neq \hat{f}^{\prime}$.

Now assume that $i>0 *$, and let $\hat{f} \in Q_{n}$ be a transformation of rank $i-1$. Once again by Lemma 3.4, it follows that $\theta^{\prime}$, and therefore $\theta$, contains $\left((\hat{f}, g),\left(\hat{f}, g^{\prime}\right)\right)$. Let $X^{\prime}$ be the dlock containing $D_{i-1, j}$, then $\left((\hat{f}, g),\left(\hat{f}, g^{\prime}\right)\right) \in \theta \cap X^{\prime 2}$. It follows that $X^{\prime}$ must be of a type for which $\pi_{2}\left(\theta \cap X^{\prime 2}\right)$ is not the identity. The six listed types in the statement of the theorem are exactly those for which this condition is satisfied.

Now $\left((\hat{f}, g),\left(\hat{f}, g^{\prime}\right)\right)=\left((\hat{f}, g),\left(\hat{f} \cdot \operatorname{id}_{S_{i-1}}, g \cdot \sigma\right)\right) \in \theta$. In the fourth case, i.e. when $X^{\prime}$ is a dlock of type $F \mathcal{H}$ with normal subgroup $\bar{N}$, we have that $\sigma \in \bar{N}$. Similarly in the fifth and sixth cases, we get that $\left(\operatorname{id}_{S_{i-1}}, \sigma\right) \in \bar{N}$. As $\sigma$ generates $N^{\prime}$ as a normal subgroup, the statements in the last three cases follow.

We conclude that the dlocks of type $\varepsilon \mathcal{H}$ can be placed onto the "west-facing" slopes of the landscape made up of the dlocks of type $F F, \mathcal{H F}$, or $\varepsilon F$. For any such slope the dlocks of 
type $\varepsilon \mathcal{H}$, must be "staked on the top of each other", with the initial $\varepsilon \mathcal{H}$-dlock being placed on either a "step" of the $F F-\mathcal{H} F-\varepsilon F$-landscape or on a dlock of type $\mathcal{H} \mathcal{H}$ or $F \mathcal{H}$ (see Figure 5). Symmetric statements hold for dlocks of type $\mathcal{H} \varepsilon$.

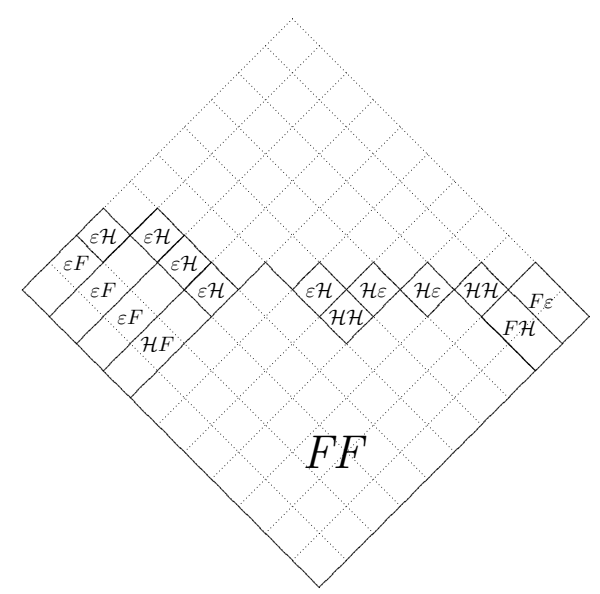

FiguRE 5. A possible configuration for dlocks of all types other than $\varepsilon \varepsilon$.

We will not derive any additional conditions for dlocks of type $\varepsilon \varepsilon$, so we may use them to fill out the remaining "spaces" in our landscape without violating any conclusion achieved so far.

The results of this section give us tight constraint about the structure of any congruence $\theta$ on $Q_{m} \times Q_{n}$. In our next theorem we will state that all the conditions we have derived so far are in fact sufficient to define a congruence.

Theorem 4.11. Let $Q \in\{\mathcal{T}, \mathcal{P} \mathcal{T}, \mathcal{I}\}$ and assume that $Q_{m}, Q_{n}$ are non-trivial. Suppose that we are given a partition $\mathcal{P}$ of $Q_{m} \times Q_{n}$ that preserves $\mathcal{D}$-classes and that to each part $B$ of $\mathcal{P}$, we associate a type from $\{F, \mathcal{H}, \varepsilon\}^{2}$ and, if the type of $B$ differs from $F F$, a group $N_{B}$. Suppose further that the following conditions are met:

(1) The partition $\mathcal{P}$ has at most one part $B$ of type $F F$, and if this is the case, then $B=D_{P}$, where $P$ is a downward-closed subset of $\{0 *, \ldots, m\} \times\{0 *, \ldots, n\}$ such that, if $Q \in\{\mathcal{I}, \mathcal{P} \mathcal{T}\}$ (in which case $0 *=0$ ),

(a) $P \neq\{0\} \times\{0, \ldots, j\}$ for all $j \in\{0, \ldots, n\}$;

(b) $P \neq\{0, \ldots, i\} \times\{0\}$ for all $i \in\{0, \ldots, m\}$.

(2) If $B$ is a part of type $\mathcal{H} F$ or $\varepsilon F$, then $B=D_{P}$ where $P$ is of the form $\{i\} \times\{0 *, \ldots, j\}$ for some $0 * \leq i \leq m, 1 \leq j \leq n$, and $N_{B} \unlhd S_{i}$. Moreover

(a) If $B$ has type $\mathcal{H} F$, then $i \geq 2$, and $N_{B} \neq \varepsilon_{i}$;

(b) If $B$ has type $\varepsilon F$, then $N_{B}=\varepsilon_{i}$.

(3) The dual of condition (2) holds for $B$ of type $F \mathcal{H}$ and $F \varepsilon$.

(4) $\mathcal{P}$ has at most one part of type $\mathcal{H} F$. If $D_{P}$, with $P=\{i\} \times\{0 *, \ldots, j\}$, is such a part, then $\mathcal{P}$ has a part $B^{\prime}$ of type $F F$, such that $D_{P^{\prime}} \subseteq B^{\prime}$, where $P^{\prime}=\{0 *, \ldots, i-1\} \times\{0 *, \ldots, j\}$.

(5) The dual of condition (4) holds for $B$ of type $F \mathcal{H}$.

(6) Let $J$ be the set of values $\left(i, j_{i}\right)$ such that the sets $\{i\} \times\left\{0 *, \ldots, j_{i}\right\}$ are exactly the index sets of the parts of $\mathcal{P}$ having type $\varepsilon F$. Then $\pi_{1}(J)$ is a set of consecutive integers (possibly empty), and the values $j_{i}$ are non-increasing in $i$.

Moreover, if $\pi(J)$ is non-empty and the smallest value $i^{\prime}$ of $\pi_{1}(J)$ is larger then $0 *$, then $\mathcal{P}$ has a part $B^{\prime}$ of type $\mathcal{H} F$ or $F F$, such that $D_{P^{\prime}} \subseteq B^{\prime}$, where $P^{\prime}=\left\{i^{\prime}-1\right\} \times$ $\left\{0 *, \ldots, j_{i^{\prime}}\right\}$.

(7) The dual of condition (6) holds for the set of $\mathcal{P}$-parts of type $F \varepsilon$.

(8) If $B$ is a part of type $\mathcal{H} \mathcal{H}, \varepsilon \mathcal{H}, \mathcal{H} \varepsilon$, or $\varepsilon \varepsilon$ then $B=D_{i, j}$ for some $0 * \leq i \leq m, 0 * \leq j \leq n$, and $N_{B} \unlhd S_{i} \times S_{j}$. Moreover,

(a) if $B$ is of type $\mathcal{H} \mathcal{H}$, then $i \geq 2, j \geq 2$, and $\pi_{1}\left(N_{B}\right) \neq \varepsilon_{i}, \pi_{2}\left(N_{B}\right) \neq \varepsilon_{j}$;

(b) if $B$ is of type $\varepsilon \mathcal{H}$, then $j \geq 2$, and $N_{B}=\varepsilon_{i} \times N^{\prime}$ for some $N^{\prime} \neq \varepsilon_{j}$;

(c) if $B$ is of type $\mathcal{H} \varepsilon$, then $i \geq 2$, and $N_{B}=N^{\prime} \times \varepsilon_{j}$ for some $N^{\prime} \neq \varepsilon_{i}$; 
(d) if $B$ is of type $\varepsilon \varepsilon$, then $N_{B}=\varepsilon_{i} \times \varepsilon_{j}$.

(9) If $B=D_{i, j}$ is a part of type $\mathcal{H} \mathcal{H}$, let $B^{\prime}$ be the part containing $D_{i, j-1}$. Then $B^{\prime}$ is either of type $F F$ or of type $\mathcal{H} F$ and $\pi_{1}\left(N_{B}\right) \subseteq N_{B^{\prime}}$. The dual condition holds for the part $B^{\prime \prime}$ containing $D_{i-1, j}$.

(10) Let $B=D_{i, j}$ be a part of type $\varepsilon \mathcal{H}$ with $N_{B}=\varepsilon_{i} \times N^{\prime}$. Let $B^{\prime}$ be the part containing $D_{i, j-1}$. Then $B^{\prime}$ is of type $F F, \mathcal{H} F$, or $\varepsilon F$.

Moreover, if $i>0 *$, the part $B^{\prime \prime}$ containing $D_{i-1, j}$ satisfies one of the following conditions:

(a) $B^{\prime \prime}$ is of type FF;

(b) $B^{\prime \prime}$ is of type $\mathcal{H} F$;

(c) $B^{\prime \prime}$ is of type $\varepsilon F$;

(d) $B^{\prime \prime}$ is of type $F \mathcal{H}$ and $N^{\prime} \subseteq N_{B^{\prime \prime}}$;

(e) $B^{\prime \prime}$ is of type $\mathcal{H} \mathcal{H}$ and $\varepsilon_{i-1} \times N^{\prime} \subseteq N_{B^{\prime \prime}}$;

(f) $B^{\prime \prime}$ is of type $\varepsilon \mathcal{H}$ and $\varepsilon_{i-1} \times N^{\prime} \subseteq N_{B^{\prime \prime}}$.

(11) The dual of condition (10) holds for the $\mathcal{P}$-parts of type $H \varepsilon$.

Suppose that on each $\mathcal{P}$-part $B$ we define a binary relation $\theta_{B}$ as follows:

(i) If $B$ has type $F F$ let $\theta_{B}=B^{2}$;

(ii) If $B$ has type $\mathcal{H} F$ or $\varepsilon F$, let $(f, g) \theta_{B}\left(f^{\prime}, g^{\prime}\right)$ if and only if $f \mathcal{H} f^{\prime}$ and $f^{\prime}=f \cdot \sigma$ for some $\sigma \in N_{B}$

(iii) If $B$ has type $F \mathcal{H}$ or $F \varepsilon$, let $(f, g) \theta_{B}\left(f^{\prime}, g^{\prime}\right)$ if and only if $g \mathcal{H} g^{\prime}$ and $g^{\prime}=g \cdot \sigma$ for some $\sigma \in N_{B}$

(iv) If $B$ has type $\mathcal{H} \mathcal{H}, \varepsilon \mathcal{H}, \mathcal{H} \varepsilon$, or $\varepsilon \varepsilon$, let $(f, g) \theta_{B}\left(f^{\prime}, g^{\prime}\right)$ if and only if $f \mathcal{H} f^{\prime}, g \mathcal{H} g^{\prime}$ and $\left(f^{\prime}, g^{\prime}\right)=(f \cdot \sigma, g \cdot \tau)$ for some $(\sigma, \tau) \in N_{B}$.

Let $\theta=\cup_{B \in \mathcal{P}} \theta_{B}$. Then $\theta$ is a congruence on $Q_{m} \times Q_{n}$.

Conversely, every congruence on $Q_{m} \times Q_{n}$ can be obtained in this way.

Proof. The "converse" part of this last theorem follows from Lemmas 4.3 to 4.10 and, where applicable, their dual versions. To show that $\theta$ is a congruence involves checking for each $\left((f, g),\left(f^{\prime}, g^{\prime}\right)\right) \in \theta$, that the principal congruence generated by $\left((f, g),\left(f^{\prime}, g^{\prime}\right)\right)$ is contained in $\theta$, using our results on principal congruences from Section 3. The proof is straightforward, but it requires the verification of many different cases. We have opted not to write it here to limit the length of the article.

Observation 4.12. We remark that Theorem 4.11 also holds for the more general case of semigroups of the form $Q_{m} \times P_{n}$, where $Q, P \in\{\mathcal{P} \mathcal{T}, \mathcal{T}, \mathcal{I}\}$, provided that the expression $0 *$ is interpreted in the context of the relevant factor and the exceptional cases (1)(a) and (1)(b) are are conditional on individual factor types. In fact, nearly all our results and proofs carry over to the case of $Q_{m} \times P_{n}$ without any other adjustments. The exceptions are Lemma 3.3, Theorem 3.7, and Theorem 3.11, which require simple and straightforward modifications.

\section{Products of three transformation semigroups}

As said above, the results of this paper essentially solve the problem of describing the congruences of $Q_{n_{1}} \times Q_{n_{2}} \times Q_{n_{3}} \ldots \times Q_{n_{k}}$, the product of finitely many transformation semigroups of the types considered, although the resulting description of the congruences would require heavy statements and notation, but not much added value. To illustrate our point, we have included a series of figures that give an idea of how the dlock-structure of a triple product looks like.

In the following figures, each $\mathcal{D}$-class is represented by a cube, and $\mathcal{D}$-classes belonging to the same dlock are combined into a colour-coded polytope. The figure is orientated so that the cube representing the $\mathcal{D}$-class of $D_{0 *, 0 *, 0 *}$ is furthest away from the observer and obstructed from view.

To reduce the number of required colors, types that are obtained by a permutation of the coordinates have the same colour. Each figure adds the dlocks from one such colour group to the previous figure. For example, Figure 6 contains one grey dlock of type $F F F$, while Figure 7 adds three red dlocks of types $F F \mathcal{H}, F \mathcal{H} F$ and $\mathcal{H F F}$. 
The following pairs of figures, from the dual and triple product case, can be considered to be in correspondence with each other: (Figure 1, Figure 6), (Figure 3, Figure 11), (Figure 4, Figure 12), and (Figure 5, Figure 14). In the remaining cases, no such direct correspondence exist due to the extra dlock-types present in the three product case.

To obtain a final configuration from Figure 14, one needs to fill out all remaining spaces with cubes that represent dlock-type $\varepsilon \varepsilon \varepsilon$. Put together, the figures demonstrate a large number, but not all, of the possible configuration of dlock-types.

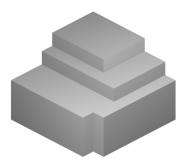

Figure $6 . F, F, F$

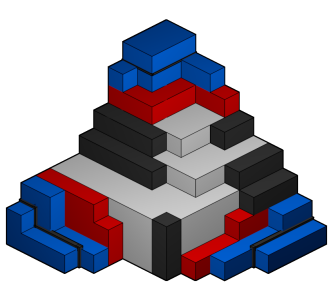

Figure 9. $F, F, \varepsilon$

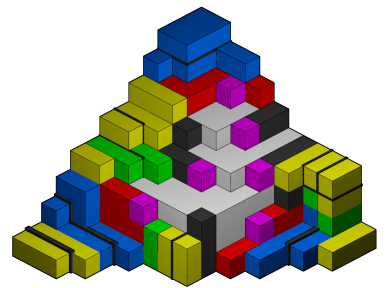

Figure $12 . \mathcal{H}, \mathcal{H}, \mathcal{H}$

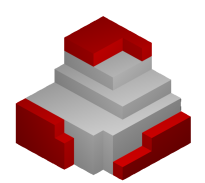

Figure 7. $F, F, \mathcal{H}$

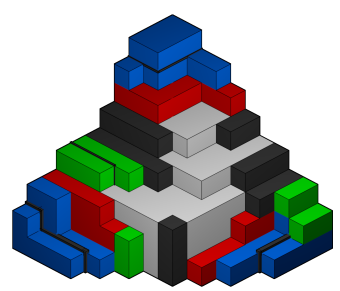

Figure 10. $F, \mathcal{H}, \varepsilon$

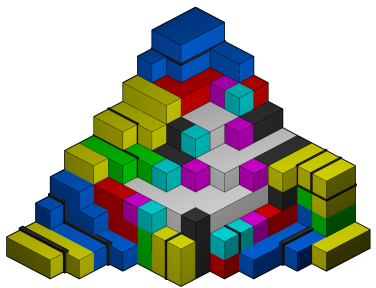

Figure 13. $\mathcal{H}, \mathcal{H}, \varepsilon$

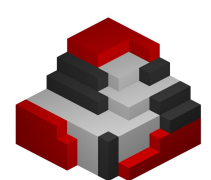

Figure 8. $F, \mathcal{H}, \mathcal{H}$

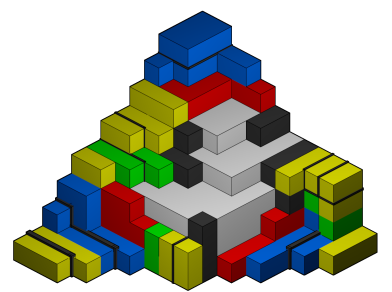

Figure 11. $F, \varepsilon, \varepsilon$

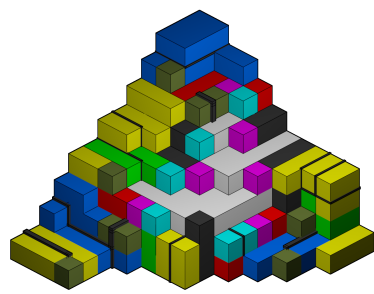

FiguRE $14 . \mathcal{H}, \varepsilon, \varepsilon$

\section{MATRIX MONOIDS}

Let $F$ be a field with multiplicative unit group $F^{*}$. Consider the multiplicative monoid $F_{n}$ of all $n \times n$-matrices over $F$. We will, throughout, identify matrices with their induced (left) linear transformation on the vector space $F^{n}$. The rank, kernel, and image of a matrix are now defined with regard to their usual meanings from linear algebra. Note that in particular the definition of kernel is now different from the notation of kernel used in the section on transformation semigroups. In addition, matrix multiplication corresponds to a composition of linear transformations that is left-to right, and hence inverse from the situation for transformation monoids.

In this section, we will determine the principal congruences on the monoid $F_{m} \times F_{n}$. As it turns out, this case closely mirrors the situation of the semigroup $\mathcal{P} \mathcal{T}_{m} \times \mathcal{P} \mathcal{T}_{n}$. In many cases, transferring the proofs of the previous sections to our new setting requires only an adaptation of notation. In those cases, we will leave it to the reader to make the relevant changes.

Other than notional changes, the main difference from the situation on $\mathcal{P} \mathcal{T}_{m} \times \mathcal{P} \mathcal{T}_{n}$ corresponds to the description of the congruence generated by a pair of the form $\left((f, g),\left(f, g^{\prime}\right)\right)$. For matrix monoids, this congruence properly relates to the congruence generated by some $\left((A, B),\left(\lambda A, B^{\prime}\right)\right)$, where $\lambda \in F^{*}$. Hence our description needs to be adapted to take care of the extra parameter $\lambda$. 
We will start by recalling several facts about the monoids $F_{n}$. Recall that two matrices are $\mathcal{R}$-related if they have the same image, $\mathcal{L}$-related if they have the same kernel, $\mathcal{H}$-related if they have the same image and kernel, and $\mathcal{D}$-related if they have the same rank.

We let $e_{i, j}$, for $1 \leq i, j \leq n$, be the elements of the standard linear basis of $F_{n}$ and set $E_{i}=e_{1,1}+e_{2,2}+\cdots+e_{i, i}$. We identify the linear group $\mathrm{GL}(i, F)$ with the maximal subgroup of $F_{n}$ that contains $E_{i}$. Denote the identity matrix on $F_{n}$ by 1 and the zero matrix by 0 . We have $1=E_{n}$, and we set $E_{0}=0$.

The description of the congruences of $F_{n}$ can be found in [34]. We will however use the following slightly different description from [27]. While this is an unpublished source, the two characterization only differ on condition (b) of the following description, and it is an easy exercise to check that they are indeed equivalent.

Theorem 6.1. A binary non-universal relation $R$ on $F_{n}$ is a congruence if and only if, there exists $\mu \in\{0, \ldots, n\}$ and

(1) there exists a normal subgroup $\bar{G}_{\mu}$ of $\mathrm{GL}(\mu, F)$;

(2) if $\mu \leq n-1$ there exist subgroups $G_{\mu+1}, G_{\mu+2}, \ldots, G_{n}$ of $F^{*}$ such that $G_{n} \subseteq G_{n-1} \subseteq$ $\ldots \subseteq G_{\mu+1}$ and $G_{\mu+1} E_{\mu} \subseteq \bar{G}_{\mu}$;

(3) two matrices $A$ and $B$ are in $R$ if and only if one of the following conditions holds:

(a) $\operatorname{rank} A<\mu$ and $\operatorname{rank} B<\mu$;

(b) $\operatorname{rank} A=\operatorname{rank} B=\mu$, and there exist $s_{1}, s_{2} \in \mathrm{GL}(n, F)$ such that $s_{1} A s_{2}$ and $s_{1} B s_{2}$ are both in $\mathrm{GL}(\mu, F)$, and belong to the same coset of $\mathrm{GL}(\mu, F)$ modulo $\bar{G}_{\mu}$;

(c) $\operatorname{rank} A=\operatorname{rank} B=i$, for some $\mu<i \leq n$, and $A=\lambda B$ for some $\lambda \in G_{i}$.

In addition, we need the following result from [27].

Lemma 6.2. A matrix $A \in F_{n}$ is a non-zero scalar multiple of the identity matrix if and only if $A$ fixes all subspaces of $F^{n}$ of dimension $n-1$.

The following will be applied later without further reference. Let $A, B \in F_{n}$. From Theorem 6.1 , the principal congruence of $F_{n}$ generated by $(A, B)$ corresponds to the following parameters in the theorem:

- If $A=\lambda B$ for some $\lambda \in F^{*}$, then $\mu=0, G_{n}=\cdots=G_{\text {rank } A+1}=\{1\}, G_{\text {rank } A}=\cdots=$ $G_{1}=\langle\lambda\rangle$, and $\bar{G}_{0}=\{0\}$

- If $\operatorname{rank} A=\operatorname{rank} B, A \neq \lambda B$ for all $\lambda \in F^{*}$, and $A \mathcal{H} B$, then $\mu=\operatorname{rank} A, G_{n}=$ $\cdots=G_{\mu+1}=\{1\}$, and $\bar{G}_{\mu}$ is the normal subgroup of $\operatorname{GL}(\mu, F)$ that corresponds to the congruence generated by the pair $\left(s_{1} A s_{2}, s_{1} B s_{2}\right)$, where $s_{1}, s_{2} \in \mathrm{GL}(n, F)$ are such that $s_{1} A s_{2}, s_{1} B s_{2} \in \mathrm{GL}(\operatorname{rank} A, F)$;

- If $(A, B) \notin \mathcal{H}$, and $\max \{\operatorname{rank} A, \operatorname{rank} B\} \leq n-1, t$ then $\mu=\max \{\operatorname{rank} A, \operatorname{rank} B\}+1$, $G_{n}=\cdots=G_{\mu+1}=\{1\}$, and $\bar{G}_{\mu}=\left\{E_{\mu}\right\}$.

- If $(A, B) \notin \mathcal{H}$ and $\max \{\operatorname{rank} A, \operatorname{rank} B\}=n$, then $(A, B)$ generates the universal congruence.

For $0 \leq i \leq m$, let $I_{i}^{(n)}$ stand for the ideal of $F_{n}$ consisting of all matrices $A$ with $\operatorname{rank} A \leq i$. We will usually just write $I_{i}$ if $n$ is deducible from the context. Let $\theta_{I_{i}}$ stand for the Rees congruence on $F_{n}$ defined by the ideal $I_{i}$.

\section{PRincipal CONGRUENCES ON $F_{m} \times F_{n}$}

For $A \in F_{m} \cup F_{n}$, let $|A|=\operatorname{rank} A$, and for $(A, B) \in F_{m} \times F_{n}$ let $|(A, B)|=(|A|,|B|)$, where we order these pairs according to the partial order $\leq \times \leq$. Throughout, $\pi_{1}$ and $\pi_{2}$ denote the projections from $F_{m} \times F_{n}$ to the first and second factor, respectively.

We will start with some general lemmas concerning congruences on $F_{m} \times F_{n}$.

Lemma 7.1. Let $\theta$ be a congruence on $F_{m} \times F_{n}$ and fix $A \in Q_{m}$; let

$$
\theta_{A}:=\left\{\left(B, B^{\prime}\right) \in F_{n} \times F_{n} \mid(A, B) \theta\left(A, B^{\prime}\right)\right\} .
$$

Then

(1) $\theta_{A}$ is a congruence on $F_{n}$; 
(2) if $A^{\prime} \in F_{m}$ and $\left|A^{\prime}\right| \leq|A|$, then $\theta_{A} \subseteq \theta_{A^{\prime}}$;

(3) if $|A|=\left|A^{\prime}\right|$, then $\theta_{A}=\theta_{A^{\prime}}$.

Proof. The proof of this lemma is virtual identical to the proof of Lemma 3.1, and is obtained from it by the syntactic substitutions $f \rightarrow A, g \rightarrow B, Q \rightarrow F$.

In an analogous construction, given a congruence $\theta$ on $F_{m} \times F_{n}$ and fixed $B \in F_{n}$, we define $\theta_{B}:=\left\{\left(A, A^{\prime}\right) \in F_{m} \times F_{m} \mid(A, B) \theta\left(A^{\prime}, B\right)\right\}$. The next result describes the ideals of $F_{n} \times F_{m}$.

Lemma 7.2. The ideals of $F_{m} \times F_{n}$ are exactly the unions of sets of the form $I_{i}^{(m)} \times I_{j}^{(n)}$, where $I_{i}^{(m)}$ and $I_{j}^{(n)}$ are ideals of $F_{m}$ and $F_{n}$, respectively.

Once again, a proof for this result can be obtained from Lemma 3.2 by making obvious adaptations. The same holds for our next result, we only have to modify parts (1) and (3) of Lemma 3.3, using the fact that $F_{m} \times F_{n}$ contains a zero element as in the case that $Q \in\{\mathcal{P} \mathcal{T}, \mathcal{I}\}$.

Lemma 7.3. Let $\theta$ be a congruence on $F_{m} \times F_{n}$. Then

(1) $\theta$ contains a class $I_{\theta}$ which is an ideal;

(2) $\theta$ contains at most one ideal class.

For the remainder of this section we fix the following notation. Let $K, K^{\prime} \in F_{m}$ and $L, L^{\prime} \in$ $F_{n}$. Let $\theta$ be a principal congruence on $F_{m} \times F_{n}$ generated by $\left((K, L),\left(K^{\prime}, L^{\prime}\right)\right)$. Let $\theta_{1}$ be the principal congruence generated by $\left(K, K^{\prime}\right)$ on $F_{m}$ and $\theta_{2}$ be the principal congruence generated by $\left(L, L^{\prime}\right)$ on $F_{n}$.

Now suppose that $(K, L),\left(K^{\prime}, L^{\prime}\right) \in F_{m} \times F_{n}$ are such that $K \mathcal{H} K^{\prime}$ and $L \mathcal{H} L^{\prime}$. Let $|K|=$ $i,|L|=j$. To the pair $\left((K, L),\left(K^{\prime}, L^{\prime}\right)\right)$ we associate a normal subgroup $H$ of $\operatorname{GL}(i, F) \times$ $\operatorname{GL}(j, F)$ as follows. As $|K|=i$ and $|L|=j$, there exist $\left(s_{1}, s_{2}\right),\left(s_{3}, s_{4}\right) \in S_{m} \times S_{n}$ such that $\left(s_{1}, s_{2}\right)(K, L)\left(s_{3}, s_{4}\right)=\left(E_{i}, E_{j}\right)$. Then $\left(s_{1}, s_{2}\right)\left(K^{\prime}, L^{\prime}\right)\left(s_{3}, s_{4}\right) \in \mathrm{GL}(i, F) \times \mathrm{GL}(j, F)$, and we take $H$ as the the normal subgroup generated by $\left(s_{1}, s_{2}\right)\left(K^{\prime}, L^{\prime}\right)\left(s_{3}, s_{4}\right)$.

We claim that the definition of $H$ is independent of our choice for $\left(s_{1}, s_{2}\right),\left(s_{3}, s_{4}\right)$. For suppose that $\left(t_{1}, t_{2}\right),\left(t_{3}, t_{4}\right) \in S_{m} \times S_{n}$ are such that $\left(t_{1}, t_{2}\right)(K, L)\left(t_{3}, t_{4}\right)=\left(E_{i}, E_{j}\right)$. Then

$$
\left(E_{i}, E_{j}\right)=\left(t_{1}, t_{2}\right)\left(s_{1}, s_{2}\right)^{-1}\left(E_{i}, E_{j}\right)\left(s_{3}, s_{4}\right)^{-1}\left(t_{3}, t_{4}\right) \text {. }
$$

Let $V, W$ be the subspaces of $F^{m}$ and $F^{n}$ generated by the columns of $E_{i}$ and $E_{j}$, respectively. It is easy to check that left multiplication by $\left(t_{1}, t_{2}\right)\left(s_{1}, s_{2}\right)^{-1}$ maps $V \times W$ onto itself. Hence there is $s \in \mathrm{GL}(i, F) \times \mathrm{GL}(j, F)$ such that for all $v \in V \times W$ we have $s v=\left(t_{1}, t_{2}\right)\left(s_{1}, s_{2}\right)^{-1} v$. It follows that $s A=\left(t_{1}, t_{2}\right)\left(s_{1}, s_{2}\right)^{-1} A$ for all $A \in \mathrm{GL}(i, F) \times \mathrm{GL}(j, F)$.

Symmetrically, there exists $t \in \mathrm{GL}(i, F) \times \mathrm{GL}(j, F)$ such that $A t=A\left(s_{3}, s_{4}\right)^{-1}\left(t_{3}, t_{4}\right)$, for all $A \in \mathrm{GL}(i, F) \times \mathrm{GL}(j, F)$. Now, (5) shows that in the group $\mathrm{GL}(i, F) \times \mathrm{GL}(j, F)$, we have $t=s^{-1}$. Thus, in $\operatorname{GL}(i, F) \times \operatorname{GL}(j, F),\left(t_{1}, t_{2}\right)\left(K^{\prime}, L^{\prime}\right)\left(t_{3}, t_{4}\right)=s\left(s_{1}, s_{2}\right)\left(K^{\prime}, L^{\prime}\right)\left(s_{3}, s_{4}\right) s^{-1}$ is a conjugate of $\left(s_{1}, s_{2}\right)\left(K^{\prime}, L^{\prime}\right)\left(s_{3}, s_{4}\right)$ and thus generates the same normal subgroup.

If $K^{\prime}=\lambda K$ for some $\lambda \in F^{*}$, it is easy to see that the normal subgroup $H$ of $\operatorname{GL}(i, F) \times$ $\mathrm{GL}(j, F)$ associated with $\left((K, L),\left(K^{\prime}, L^{\prime}\right)\right)$ is contained in $F^{*} E_{i} \times \mathrm{GL}(j, F)$. We then associate a normal subgroup $\hat{H}$ of $F^{*} \times \mathrm{GL}(j, F)$ with the pair $\left((K, L),\left(K^{\prime}, L^{\prime}\right)\right)$, taking $\hat{H}$ as the image of $H$ under the canonical map from $F^{*} E_{i} \times \mathrm{GL}(j, F)$ to $F^{*} \times \mathrm{GL}(j, F)$. If $L^{\prime}=\lambda L$ for some $\lambda \in F^{*}$, we dually associate a normal subgroup of $\mathrm{GL}(i, F) \times F^{*}$.

Lemma 7.4. Let $\theta$ be a congruence on $F_{m} \times F_{n}$. Let $(K, L),\left(K^{\prime}, L^{\prime}\right) \in F_{m} \times F_{n}$ be such that $(K, L) \theta\left(K^{\prime}, L^{\prime}\right), K \mathcal{H} K^{\prime}$, and $L \mathcal{H} L^{\prime}$. Let $|K|=i,|L|=j$, and $H$ be the normal subgroup of $\mathrm{GL}(i, F) \times \mathrm{GL}(j, F)$ associated with the pair $\left((K, L),\left(K^{\prime}, L^{\prime}\right)\right)$.

Let $(M, N),\left(M, N^{\prime}\right) \in F_{m} \times F_{n}$ where $|M|=i,|N|=j, M \mathcal{H} M^{\prime}$ and $N \mathcal{H} N^{\prime}$. Let $H^{\prime}$ be the normal subgroup of $\mathrm{GL}(i, F) \times \mathrm{GL}(j, F)$ associated with the pair $\left((M, N),\left(M^{\prime}, N^{\prime}\right)\right)$. If $H^{\prime} \subseteq H$, then $(M, N) \theta\left(M^{\prime}, N^{\prime}\right)$.

Proof. Let $\left(s_{1}, s_{2}\right),\left(s_{3}, s_{4}\right) \in S_{m} \times S_{n}$ be the elements considered in the definition of $H$ : $\left(s_{1}, s_{2}\right)(K, L)\left(s_{3}, s_{4}\right)=\left(E_{i}, E_{j}\right)$ and $H$ is the normal subgroup of $\mathrm{GL}(i, F) \times \mathrm{GL}(j, F)$ generated by $\left(s_{1}, s_{2}\right)\left(K^{\prime}, L^{\prime}\right)\left(s_{3}, s_{4}\right)$. Let $\left(t_{1}, t_{2}\right),\left(t_{3}, t_{4}\right) \in S_{m} \times S_{n}$ be the corresponding elements 
taken to define $H^{\prime}$. We have that

$$
\left(s_{1}, s_{2}\right)\left(K^{\prime}, L^{\prime}\right)\left(s_{3}, s_{4}\right) \theta\left(s_{1}, s_{2}\right)(K, L)\left(s_{3}, s_{4}\right)=\left(E_{i}, E_{j}\right) .
$$

Let $\hat{\theta}$ be the restriction of $\theta$ to the group $\operatorname{GL}(i, F) \times \mathrm{GL}(j, F)$, and $R$ the normal subgroup of $\mathrm{GL}(i, F) \times \mathrm{GL}(j, F)$ corresponding to $\hat{\theta}$. By $(6)$, we have $\left(s_{1}, s_{2}\right)\left(K^{\prime}, L^{\prime}\right)\left(s_{3}, s_{4}\right) \in R$. As $H$ is normal, $H \subseteq R$, and so $H^{\prime} \subseteq R$, since $H^{\prime} \subseteq H$. In particular, $\left(t_{1}, t_{2}\right)\left(M^{\prime}, N^{\prime}\right)\left(t_{3}, t_{4}\right) \in R$, hence $\left(t_{1}, t_{2}\right)\left(M^{\prime}, N^{\prime}\right)\left(t_{3}, t_{4}\right) \theta\left(E_{i}, E_{j}\right)$, and thus

$$
(M, N)=\left(t_{1}, t_{2}\right)^{-1}\left(E_{i}, E_{j}\right)\left(t_{3}, t_{4}\right)^{-1} \theta\left(t_{1}, t_{2}\right)^{-1}\left(\left(t_{1}, t_{2}\right)\left(M^{\prime}, N^{\prime}\right)\left(t_{3}, t_{4}\right)\right)\left(t_{3}, t_{4}\right)^{-1}=\left(M^{\prime}, N^{\prime}\right) .
$$

Lemma 7.5. Let $\theta$ be a congruence on $F_{m} \times F_{n}$. Let $(K, L),\left(K^{\prime}, L^{\prime}\right) \in F_{m} \times F_{n}$ be such that $(K, L) \theta\left(K^{\prime}, L^{\prime}\right), L \mathcal{H} L^{\prime}$, and $K^{\prime}=\lambda K$ for some $\lambda \in F^{*}$. Let $|K|=i,|L|=j$, and $\hat{H}$ be the normal subgroup of $F^{*} \times \mathrm{GL}(j, F)$ associated with the pair $\left((K, L),\left(K^{\prime}, L^{\prime}\right)\right)$.

Suppose that $(M, N),\left(M, N^{\prime}\right) \in F_{m} \times F_{n}$ are such that $|M|=k \leq i,|N|=j, N \mathcal{H} N^{\prime}$, and $M^{\prime}=\lambda^{\prime} M$ for some $\lambda^{\prime} \in F^{*}$. Let $\hat{H}^{\prime}$ be the normal subgroup of $F^{*} \times \operatorname{GL}(j, F)$ associated with the pair $\left((M, N),\left(M^{\prime}, N^{\prime}\right)\right)$. If $\hat{H}^{\prime} \subseteq \hat{H}$, then $(M, N) \theta\left(M^{\prime}, N^{\prime}\right)$.

Proof. We have $K \mathcal{H} K^{\prime}$ and $M \mathcal{H} M^{\prime}$ so, by Lemma 7.4, we may assume that $(K, L)=\left(E_{i}, E_{j}\right)$. We then have $\left(E_{i}, E_{j}\right) \theta\left(\lambda E_{i}, L^{\prime}\right)$, and $\left(E_{k}, E_{j}\right)=\left(E_{k}, E_{n}\right)\left(E_{i}, E_{j}\right) \theta\left(E_{k}, E_{n}\right)\left(\lambda E_{i}, L^{\prime}\right)=\left(\lambda E_{k}, L^{\prime}\right)$. It is straightforward to check that the normal subgroup of $F^{*} \times \operatorname{GL}(k, F)$ associated with $\left(\left(E_{k}, E_{j}\right),\left(\lambda E_{k}, L^{\prime}\right)\right)$ is $\hat{H}$.

Let $\bar{H}$ be the normal subgroup of $\operatorname{GL}(k, F) \times \mathrm{GL}(j, F)$ associated with this pair, so that $\hat{H}=\phi(\bar{H})$, where $\phi$ is the natural isomorphism from $F^{*} E_{k} \times \operatorname{GL}(j, F)$ to $F^{*} \times \operatorname{GL}(j, F)$. Let $\bar{H}^{\prime}$ be the normal subgroup of $\mathrm{GL}(k, F) \times \mathrm{GL}(j, F)$ associated with the pair $\left((M, N),\left(M^{\prime}, N^{\prime}\right)\right)$. Notice that $\bar{H}^{\prime} \subseteq F^{*} E_{k} \times \operatorname{GL}(j, F)$ since $M^{\prime}=\lambda^{\prime} M$. Then $\bar{H}^{\prime}=\phi^{-1}\left(\hat{H}^{\prime}\right) \subseteq \phi^{-1}(\hat{H})=\bar{H}$. The result now follows with Lemma 7.4.

It is clear that a dual version of Lemma 7.5 holds as well, so let us look at the principal congruences on $F_{m} \times F_{n}$.

Theorem 7.6. Let $\theta$ be a principal congruence on $F_{m} \times F_{n}$ generated by $\left((K, L),\left(K^{\prime}, L^{\prime}\right)\right)$. If $K^{\prime}=\lambda K$ and $L^{\prime}=\nu L$ for some $\lambda, \nu \in F^{*}$, we have $(M, N) \theta\left(M^{\prime}, N^{\prime}\right)$ if and only if $(M, N)=$ $\left(M^{\prime}, N^{\prime}\right)$ or $|M| \leq|K|,|N| \leq|L|$, and there exists a $\left(\lambda^{\prime}, \nu^{\prime}\right) \in\langle(\lambda, \nu)\rangle \subseteq F^{*} \times F^{*}$ such that $M^{\prime}=\lambda^{\prime} M$ and $N^{\prime}=\nu^{\prime} N$.

Proof. This theorem can be shown by applying Lemma 7.5 followed by its dual. However, we will give a short direct proof.

Let $\theta^{\prime}$ be the binary relation defined by the statement of this theorem.

If $|M| \leq|K|,|N| \leq|L|$ then $M=U K V, N=W L X$ for some $U, V \in F_{m}, W, X \in F_{n}$, and $(M, N)=(U K V, W L X) \theta\left(U K^{\prime} V, W L^{\prime} X\right)=(U \lambda K V, W \nu L X)=(\lambda U K V, \nu W L X)=(\lambda M, \nu N)$. As $|M| \leq|K|=\left|K^{\prime}\right|,|N| \leq|L|=\left|L^{\prime}\right|$, we can similarly show that $(M, N) \theta\left(\lambda^{-1} M, \nu^{-1} N\right)$. Thus $\theta^{\prime} \subseteq \theta$, since $\left(M^{\prime}, N^{\prime}\right)=\left(\lambda^{\prime} M, \nu^{\prime} N\right)$, and $\left(\lambda^{\prime}, \nu^{\prime}\right)$ belongs to the cyclic group generated by $(\lambda, \nu)$.

Conversely, it is straightforward to check that $\theta^{\prime}$ is a congruence containing $\left((K, L),\left(K^{\prime}, L^{\prime}\right)\right)$, therefore $\theta \subseteq \theta^{\prime}$. The result follows.

Lemma 7.7. Let $\theta$ be the principal congruence on $F_{m} \times F_{n}$ generated by $\left((K, L),\left(K^{\prime}, L^{\prime}\right)\right)$, and let $j=\max \left\{|K|,\left|K^{\prime}\right|\right\}$. If $\left(K, K^{\prime}\right) \notin \mathcal{H}$, then $\theta_{L}$ or $\theta_{L^{\prime}}$ contains the Rees congruence $\theta_{I_{j}}$ of $F_{m}$.

We remark that the following proof is essentially equivalent to the proof of Lemma 3.6. As the technical adaptations required to transform one lemma into the other are more complex than in previous cases, we have decided to provide a complete proof.

Proof. We will show that for $j=\left|K^{\prime}\right|, \theta_{I_{j}} \subseteq \theta_{L^{\prime}}$. An anologous result for $j=|K|$ follows symmetrically. So let us assume that $|K| \leq\left|K^{\prime}\right|=j$. As $\left(K, K^{\prime}\right) \notin \mathcal{H}, K$ and $K^{\prime}$ must differ in either the image or the kernel. We consider two cases.

First case: $\operatorname{im} K \neq \operatorname{im} K^{\prime}$. 
As $\left|K^{\prime}\right|=j \geq|K|$, then $\operatorname{im} K^{\prime} \nsubseteq \operatorname{im} K$. As $F_{m}$ is regular, there exists an idempotent $M \in F_{m}$ such that $M \mathcal{R} K$. Hence $\operatorname{im} M=\operatorname{im} K$, and as $M$ is idempotent, $M K=K$.

We have that $\left(M K^{\prime}, L^{\prime}\right)=\left(M, E_{n}\right)\left(K^{\prime}, L^{\prime}\right) \theta\left(M, E_{n}\right)(K, L)=(M K, L)=(K, L) \theta\left(K^{\prime}, L^{\prime}\right)$ and so $\left(M K^{\prime}, K^{\prime}\right) \in \theta_{L^{\prime}}$ on $F_{m}$. As $\operatorname{im} K^{\prime} \nsubseteq \operatorname{im} K$, and $\operatorname{im} M=\operatorname{im} K$, the transformations $M K^{\prime}$ and $K^{\prime}$ have different images, and it follows that $\left(M K^{\prime}, K^{\prime}\right) \notin \mathcal{H}$. Now, the congruence $\theta^{\prime}$ generated by $\left(M K^{\prime}, K^{\prime}\right)$ is contained in $\theta_{L^{\prime}}$ and by Theorem 6.1 and the remarks following it, we have $\theta^{\prime}=\theta_{I_{j}}$. We get $\theta_{I_{j}} \subseteq \theta_{L^{\prime}}$.

Second case: $\operatorname{ker} K \neq \operatorname{ker} K^{\prime}$.

Now $\left|K^{\prime}\right|=j \geq|K|$, implies that $\operatorname{ker} K \nsubseteq \mathbb{\text { ker }} K^{\prime}$. As above, the regularity of $F_{m}$ implies that there exists an idempotent $M$ that is $\mathcal{L}$-related to $K$; thus $M$ and $K$ have the same kernel and $K M=K$. Hence $\left(K^{\prime} M, L^{\prime}\right)=\left(K^{\prime}, L^{\prime}\right)\left(M, E_{n}\right) \theta(K, L)\left(M, E_{n}\right)=(K M, L)=(K, L) \theta\left(K^{\prime}, L^{\prime}\right)$ and so $\left(K^{\prime} M, K^{\prime}\right) \in \theta_{L^{\prime}}$. Now $\operatorname{ker} K=\operatorname{ker} M \subseteq \operatorname{ker}\left(K^{\prime} M\right)$. As $\operatorname{ker} K \nsubseteq \subseteq \operatorname{ker} K^{\prime}$, we get $\operatorname{ker} K^{\prime} \neq \operatorname{ker}\left(K^{\prime} M\right)$ and so $\left(K^{\prime} M, K^{\prime}\right) \notin \mathcal{H}$. As above, by Theorem 6.1 and the remarks following it, we get $\theta_{I_{j}} \subseteq \theta_{L^{\prime}}$.

Theorem 7.8. Let $\theta$ be the congruence on $F_{m} \times F_{n}$ generated by $\left((K, L),\left(K^{\prime}, L^{\prime}\right)\right)$. Assume that $\left(K, K^{\prime}\right) \notin \mathcal{H},\left(L, L^{\prime}\right) \notin \mathcal{H},|K|=i,\left|K^{\prime}\right|=j,|L|=k$, and $\left|L^{\prime}\right|=l$. Then $\theta$ is the Rees congruence on $F_{m} \times F_{n}$ defined by the ideal $J=I_{i} \times I_{k} \cup I_{j} \times I_{l}$.

The proof of this theorem is once again essentially the proof of Theorem 3.7.

Corollary 7.9. Under the conditions of Theorem 7.8, if $i \leq j$ and $k \leq l$ then $\theta=\theta_{I_{j} \times I_{l}}$.

The proof of the following Theorem corresponds to the proof of Theorem 3.9.

Theorem 7.10. Let $\theta$ be the congruence on $F_{m} \times F_{n}$ generated by $\left((K, L),\left(K^{\prime}, L^{\prime}\right)\right)$, and let $\theta_{2}$ be the congruence on $F_{n}$ generated by $\left(L, L^{\prime}\right)$. If $\left(L, L^{\prime}\right) \in \mathcal{H}$ and $\left(K, K^{\prime}\right) \notin \mathcal{H}$; let $j=$ $\max \left\{|K|,\left|K^{\prime}\right|\right\}$ and $k=|L|=\left|L^{\prime}\right|$. Then $(M, N) \theta\left(M^{\prime}, N^{\prime}\right)$ if and only if $(M, N)=\left(M^{\prime}, N^{\prime}\right)$ or $|M|,\left|M^{\prime}\right| \leq j,|N|,\left|N^{\prime}\right| \leq k, N \theta_{2} N^{\prime}$.

Notice that we can once again give a more explicit description of $\theta$ by incorporating the classification of $\theta_{2}$ given by Theorem 6.1 .

Corollary 7.11. (A) Let $(K, L),\left(K^{\prime}, L^{\prime}\right) \in F_{m} \times F_{n}$, such that $L^{\prime} \neq \lambda L$ for any $\lambda \in F^{*}$, $\left(L, L^{\prime}\right) \in \mathcal{H}$ and $\left(K, K^{\prime}\right) \notin \mathcal{H}$. Let $j=\max \left\{|K|,\left|K^{\prime}\right|\right\}$ and $k=|L|=\left|L^{\prime}\right|$. Let $s_{1}, s_{2} \in \mathrm{GL}(n, F)$ be such that $s_{1} L s_{2}$ and $s_{1} L^{\prime} s_{2}$ are in $\mathrm{GL}(k, F)$, and $H$ the normal subgroup of $\mathrm{GL}(k, F)$ generated by $\left(s_{1} L s_{2}, s_{1} L^{\prime} s_{2}\right)$. If $\theta$ is the congruence on $F_{m} \times F_{n}$ generated by $\left((K, L),\left(K^{\prime}, L^{\prime}\right)\right)$, then $(M, N) \theta\left(M^{\prime}, N^{\prime}\right)$ if and only if one of the following holds:

(1) $(M, N)=\left(M^{\prime}, N^{\prime}\right)$ for $|M|>j$ or $|N|>k$,

(2) $|M|,\left|M^{\prime}\right| \leq j,|N|=k, N \mathcal{H} N^{\prime}$ and there exist $t_{1}, t_{2} \in \mathrm{GL}(n, F)$ such that $t_{1} N t_{2}, t_{1} N^{\prime} t_{2} \in$ $\mathrm{GL}(k, F)$ and lie in the same coset of $H$.

(3) $|M|,\left|M^{\prime}\right| \leq j$ and $|N|,\left|N^{\prime}\right|<k$.

(B) Let $(K, L),\left(K^{\prime}, L^{\prime}\right) \in F_{m} \times F_{n}$, such that $L^{\prime}=\lambda L$ for some $\lambda \in F^{*}$, and $\left(K, K^{\prime}\right) \notin \mathcal{H}$. Let $j=\max \left\{|K|,\left|K^{\prime}\right|\right\}$ and $k=|L|=\left|L^{\prime}\right|$. Let $H$ be the subgroup of $F^{*}$ generated by $\lambda$. If $\theta$ is the congruence on $F_{m} \times F_{n}$ generated by $\left((K, L),\left(K^{\prime}, L^{\prime}\right)\right)$, then $(M, N) \theta\left(M^{\prime}, N^{\prime}\right)$ if and only if one of the following holds:

(1) $(M, N)=\left(M^{\prime}, N^{\prime}\right)$ for $|M|>j$ or $|N|>k$,

(2) $|M|,\left|M^{\prime}\right| \leq j,|N| \leq k, N^{\prime}=\lambda^{\prime} N$ for some $\lambda^{\prime} \in H$.

Switching the roles of the coordinates we get an obvious dual version of Theorem 7.10.

Theorem 7.12. Let $\theta$ be the principal congruence on $F_{m} \times F_{n}$ generated by $\left((K, L),\left(K^{\prime}, L^{\prime}\right)\right)$, where $K^{\prime}=\lambda K$ for $\lambda \in F^{*}$, and $L \mathcal{H} L^{\prime}$, such that $L^{\prime}$ is not a scalar multiple of $L$. Let $|K|=i$, $|L|=j, \hat{H}$ the the normal subgroup of $F^{*} \times \mathrm{GL}(j, F)$ associated with the pair $\left((K, L),\left(K^{\prime}, L^{\prime}\right)\right)$, and $G$ the subgroup of $F^{*}$ generated by $\lambda$. Then, for $(M, N),\left(M^{\prime}, N^{\prime}\right) \in F_{m} \times F_{n},(M, N) \theta\left(M^{\prime}, N^{\prime}\right)$ if and only if one of the following holds:

(1) $(M, N)=\left(M^{\prime}, N^{\prime}\right)$; 
(2) $|M| \leq i, M^{\prime} \mathcal{H} M,|N|=j, N^{\prime} \mathcal{H} N$ and the normal subgroup $\hat{H}^{\prime}$ of $F^{*} \times \mathrm{GL}(j, F)$ associated with the pair $\left((M, N),\left(M^{\prime}, N^{\prime}\right)\right)$ is contained in $\hat{H}$;

(3) $|M| \leq i, M^{\prime}=\lambda^{\prime} M$ for some $\lambda^{\prime} \in G,|N|,\left|N^{\prime}\right|<j$.

Proof. Let $\theta^{\prime}$ be the relation defined by the statement of the theorem. It is straightforward to check that $\theta^{\prime}$ is a congruence.

Conversely, $\theta$ contains the pairs from (1) trivially, and the ones from (2) by Lemma 7.5. It remains to show that $\theta$ contains the pairs from (3). By multiplying with suitable $\left(E_{n}, s_{1}\right)$, $\left(E_{n}, s_{2}\right) \in \mathrm{GL}(m, F) \times \mathrm{GL}(n, F)$ on the left and right, we may assume that $L=E_{j}, L^{\prime} \in$ $\mathrm{GL}(j, F)$, and that $L^{\prime}$ is not a scalar multiple of $E_{j}$. For the following considerations, we will identify the ideal $F_{n} E_{j} F_{n}$ with $F_{j}$.

Now, as $L^{\prime}$ is not a scalar multiple of $E_{j}$, by Lemma 6.2 , there exists a subspace $V$ of $F^{j}$ with dimension $j-1$ that is not preserved by $L^{\prime}$. Let $A \in F_{j}$ be such that $A$ has rank $j-1$ and is the identity on $V$. Then $\left(K^{\prime}, L^{\prime} A\right)=\left(K^{\prime}, L^{\prime}\right)\left(E_{m}, A\right) \theta(K, L)\left(E_{m}, A\right)=\left(K, E_{j} A\right)$.

Considering those elements in $F_{m} \times F_{n}$ again, we see that $V=\operatorname{im}\left(E_{j} A\right) \neq \operatorname{im}\left(L^{\prime} A\right)$, and so $\left(E_{j} A, L^{\prime} A\right) \notin \mathcal{H}$. By applying Theorem 7.10 to the pair $\left(\left(K, E_{j} A\right),\left(K^{\prime}, L^{\prime} A\right)\right)$, we see that the pairs in (3) belong to $\theta$.

In view of Theorem 7.6, Theorem 7.8, Theorem 7.10, Theorem 7.12, and, where applicable, their dual versions, it remains to determine the principal congruence $\theta$ when $K \mathcal{H} K, L \mathcal{H} L^{\prime}$, $K^{\prime} \neq \lambda K$ for all $\lambda \in F^{*}$ and $L^{\prime} \neq \lambda L$ for all $\lambda \in F^{*}$.

Theorem 7.13. Let $\theta$ be the principal congruence on $F_{m} \times F_{n}$ generated by $\left((K, L),\left(K^{\prime}, L^{\prime}\right)\right)$, where $K \mathcal{H} K^{\prime}$, and $L \mathcal{H} L^{\prime}$, such that $K^{\prime}$ is not a scalar multiple of $K$ and $L^{\prime}$ is not a scalar multiple of $L$. Let $|K|=i,|L|=j, H$ the the normal subgroup of $\mathrm{GL}(i, F) \times \mathrm{GL}(j, F)$ associated with the pair $\left((K, L),\left(K^{\prime}, L^{\prime}\right)\right)$. Then for $(M, N),\left(M^{\prime}, N^{\prime}\right) \in F_{m} \times F_{n},(M, N) \theta\left(M^{\prime}, N^{\prime}\right)$ if and only if one of the following holds:

(1) $(M, N)=\left(M^{\prime}, N^{\prime}\right)$;

(2) $|M|=i, M^{\prime} \mathcal{H} M,|N|=j, N^{\prime} \mathcal{H} N$ and the normal subgroup $H^{\prime}$ of $\mathrm{GL}(i, F) \times \operatorname{GL}(j, F)$ associated with the pair $\left((M, N),\left(M^{\prime}, N^{\prime}\right)\right)$ is contained in $H$;

(3) $|M|=i, M^{\prime} \mathcal{H} M,|N|,\left|N^{\prime}\right|<j$, and there exists $s_{1}, s_{2} \in \mathrm{GL}(m, F)$ such that $s_{1} M s_{2}$ and $s_{2} M^{\prime} s_{2}$ are both in $\mathrm{GL}(i, F)$ and belong to the same coset of $\mathrm{GL}(i, F)$ modulo $\pi_{1}(H)$;

(4) $|N|=j, N^{\prime} \mathcal{H} N,|M|,\left|M^{\prime}\right|<i$, and there exists $s_{1}, s_{2} \in \operatorname{GL}(n, F)$ such that $s_{1} N s_{2}$ and $s_{2} N^{\prime} s_{2}$ are both in $\mathrm{GL}(j, F)$ and belong to the same coset of $\mathrm{GL}(j, F)$ modulo $\pi_{2}(H)$;

(5) $|M|,\left|M^{\prime}\right|<i,|N|,\left|N^{\prime}\right|<j$.

Proof. Let $\theta^{\prime}$ be the relation defined by the statement of the theorem. It is straightforward to check that $\theta^{\prime}$ is a congruence.

Conversely, $\theta$ contains the pairs from (1) trivially, and the ones from (2) by Lemma 7.4.

It remains to show that $\theta$ contains the pairs from (3), (4), and (5). Using an analogous argument to the last part of the proof of Theorem 7.12, we can find an $A \in F_{n}$ such that $\left(K^{\prime}, L^{\prime} A\right) \theta\left(K, E_{j} A\right)$, where $L^{\prime} A$ and $E_{j} A$ have rank $j-1$ but have different images and hence are not $\mathcal{H}$-related. Now the congruence $\beta$ generated by $\left(\left(K^{\prime}, L^{\prime} A\right),\left(K, E_{j} A\right)\right)$ is contained in $\theta$ and an application of Corollary 7.11 to $\beta$ shows that $\beta$ contains all pairs from (3) and (5), and then so does $\theta$. Finally, $\theta$ contains the pairs in (4) by a symmetric argument.

\section{Problems}

In this final section we propose a number of problems motivated by the results above. Clearly, the most natural is the following.

Problem 8.1. Let $S$ and $T$ be any transformation semigroup whose congruences have been described. Find the congruences of $S \times T$.

The description of the congruences of $\mathcal{T}_{n}$ provided in [33] is used in [37] to describe the endomorphisms of $\mathcal{T}_{n}$. Regarding the monoid $F_{n}$, its congruences are known since 1953 [34], but the description of $\operatorname{End}\left(F_{n}\right)$ is still to be done. 
Problem 8.2. Describe the endomorphisms of the monoid $Q_{n} \times Q_{m}$, where $Q \in\{\mathcal{P} \mathcal{T}, \mathcal{T}, \mathcal{I}\}$, and the endomorphisms of the monoid $F_{n}$. Use the description of the congruences on $S=F_{n} \times F_{m}$ to find the endomorphisms of $S$.

Let $G$ be a permutation group contained in the symmetric group $S_{n}$ and let $t \in \mathcal{T}_{n}$; let $\langle G, t\rangle$ denote the submonoid of $T_{n}$ generated by $G$ and $t$. These monoids proved to be a source of exciting new results involving different parts of Mathematics such as group and semigroup theories, combinatorics, number theory, linear algebra or computational algebra. (For an illustration see $[2,5-11,28-31,35,36,39]$ and the references therein.) In [30], the congruences of the monoids $\left\langle S_{n}, t\right\rangle$ are described, and so in this context one may ask the following.

Problem 8.3. Let $G \leq S_{n}$ be a permutation group (in particular a 2-transitive or imprimitive group) and let $t, q \in \mathcal{T}_{n} \backslash S_{n}$. Describe the congruences and the endomorphisms of $\langle G, t\rangle$ and of $\langle G, t\rangle \times\langle G, q\rangle$.

The previous problems admit linear analogous.

Problem 8.4. Let $V$ be a finite dimension vector space and let $G \leq \operatorname{Aut}(V) ;$ let $t, q \in \operatorname{End}(V) \backslash$ $\operatorname{Aut}(V)$. Describe the congruences and the endomorphisms of $\langle G, t\rangle$ and of $\langle G, t\rangle \times\langle G, p\rangle$. For some results on linear monoids of the form $\langle G, t\rangle$ we refer the reader to [15].

The next natural step is to ask for analogous results for the endomorphism monoid of an independence algebra, as sets and vector spaces are examples of such algebras. See $[12,17,25]$.

Problem 8.5. Let $A$ and $B$ be finite dimension independence algebras. Describe the congruences on $\operatorname{End}(A) \times \operatorname{End}(B)$.

To tackle this problem one should rely on the classification of these algebras $[17,40]$ as in [4].

Clearly, the same type of questions may be posed for other algebras.

Problem 8.6. Solve the analogous problems for the algebras introduced in [22, 23] and for $M C$-algebras, $M S$-algebras, SC-algebras, and SC-ranked algebras [16, Chapter 8].

A first step towards the solution of this last problem would be to solve it for an $S C$-ranked free $M$-act and for an $S C$-ranked free module over an $\aleph_{1}$-Noetherian ring [16].

We recall that we were driven to the results in this paper by some considerations on centralizers of idempotents. Let $e^{2}=e \in \mathcal{T}_{n}$, and denote by $C_{\mathcal{T}_{n}}(e)$ the centralizer of the idempotent $e$ in $\mathcal{T}_{n}$, that is, $C_{\mathcal{T}_{n}}(e)=\left\{f \in \mathcal{T}_{n} \mid f e=e f\right\}$. The monoid $C_{\mathcal{T}_{n}}(e)$ has very interesting features, in particular it generalizes both $\mathcal{T}_{n}$ and $\mathcal{P} \mathcal{T}_{n}$. See $[3,13,14]$.

Problem 8.7. Describe the congruences of $C_{\mathcal{T}_{n}}(e)$, starting with the regular case. See [3].

The solution of this problem requires a complete description of the congruences of $\mathcal{P} \mathcal{T}_{n} \times$ $\mathcal{P} \mathcal{T}_{m}$, and that was what prompted us to write this paper.

Finally, we cannot avoid thinking about the partition monoid [1,18-21]; this has a very rich structure and has been attracting increasing attention.

Problem 8.8. Describe the congruences and the endomorphisms of the partition monoid. Solve similar problems for the direct product of two partition monoids.

\section{ACKNOWLEDGEMENTS}

The authors were supported by FCT (Portugal) through project UID/MULTI/04621/2013 of CEMAT-CIÊNCIAS.

The second author has received funding from the European Union Seventh Framework Programme (FP7/2007-2013) under grant agreement no. PCOFUND-GA-2009-246542 and from the Foundation for Science and Technology of Portugal under PCOFUND-GA-2009-246542 and $\mathrm{SFRH} / \mathrm{BCC} / 52684 / 2014$. 


\section{REFERENCES}

[1] C. Ahmed, P. Martin and Volodymyr Mazorchuk. On the number of principal ideals in d-tonal partition monoids. arXiv: 1503.06718

[2] J. André, J. Araújo and P.J. Cameron. The classification of partition homogeneous groups with applications to semigroup theory. Journal of Algebra 452, 288-310.

[3] J. M. André, J. Araújo and J. Konieczny. Regular centralizers of idempotent transformations. Semigroup Forum 82 (2) (2011), 307-318.

[4] J. Araújo, W. Bentz and J. Konieczny. The largest subsemilattices of the semigroup of endomorphisms of an independence algebra. Linear Algebra and its Applications 458 (2014), 50-79.

[5] J. Araújo, W. Bentz, P. J. Cameron, G. Royle and A. Schaefer. Primitive groups and synchronization. Proceedings of the London Mathematical Society 113 (2016), 829-867.

[6] J. Araújo, W. Bentz, J. D. Mitchell and C. Schneider. The rank of the semigroup of transformations stabilising a partition of a finite set. Mathematical Proceedings of the Cambridge Philosophical Society 159 (2) (2015), 339-353.

[7] J. Araújo, W. Bentz, E. Dobson, J. Konieczny and J. Morris. Automorphism groups of circulant digraphs with applications to semigroup theory. Combinatorica (2017), 1-28.

[8] J. Araújo and P. J. Cameron. Primitive groups synchronize non-uniform maps of extreme ranks. Journal of Combinatorial Theory, Series B, 106 (2014), 98-114.

[9] J. Araújo and P. J. Cameron. Two Generalizations of Homogeneity in Groups with Applications to Regular Semigroups. Transactions American Mathematical Society 368 (2016), 1159-1188.

[10] J. Araújo, P. J. Cameron, J. D. Mitchell, M. Neuhoffer. The classification of normalizing groups. Journal of Algebra 373 (2013), 481-490.

[11] J. Araújo, P. J. Cameron and B. Steinberg. Between primitive and 2-transitive: Synchronization and its friends. European Mathematical Society Surveys in Mathematical Sciences 4:2 (2017), 101-184.

[12] J. Araújo and J. Fountain. The origins of independence algebras. Semigroups and languages, 54-67, World Sci. Publ., River Edge, NJ, 2004.

[13] J. Araújo and J. Konieczny. Semigroups of transformations preserving an equivalence relation and a crosssection. Communications in Algebra 32 (2004), 1917-1935.

[14] J. Araújo and J. Konieczny. Centralizers in the full transformation semigroup. Semigroup Forum 86 (2013), $1-31$.

[15] J. Araújo, and F.C. Silva. Semigroups of linear endomorphisms closed under conjugation. Communications in Algebra 28 (8) (2000), 3679-3689.

[16] J. Araújo and F. Wehrung. Embedding properties of endomorphism semigroups. Fundamenta Mathematicae 202 (2009), 125-146.

[17] P. J. Cameron and C. Szabó. Independence algebras. Journal of the London Mathematical Society 61 (2000), 321-334.

[18] J. East. Generators and relations for partition monoids and algebras. Journal of Algebra 339 (2011), 1-26.

[19] J. East. On the singular part of the partition monoid. International Journal of Algebra and Computation 21 (2011), no. 1-2, 147-178.

[20] I. Dolinka, J. East, A. Evangelou, D. FitzGerald, N. Ham, J. Hyde and N. Loughlin. Enumeration of idempotents in diagram semigroups and algebras. Journal of Combinatorial Theory, Series A, 131 (2015), $119-152$.

[21] D.G. FitzGerald and K. W. Lau. On the partition monoid and some related semigroups. Bulletin of the Australian Mathematical Society 83 (2011), no. 2, 273-288.

[22] J.Fountain and V. Gould. Relatively free algebras with weak exchange properties. Journal of the Australian Mathematical Society 75 (2003), 355-384.

[23] J.Fountain and V. Gould. Endomorphisms of relatively free algebras with weak exchange properties. Algebra Universalis 51 (2004), 257-285.

[24] O. Ganyushkin and V. Mazorchuk. Classical finite transformation semigroups. An introduction. Algebra and Applications, 9. Springer-Verlag London, Ltd., London, 2009.

[25] V. Gould. Independence algebras. Algebra Universalis 33 (1995), 294-318.

[26] John M. Howie. Fundamentals of semigroup theory, volume 12 of London Mathematical Society Monographs. New Series. The Clarendon Press Oxford University Press, New York, 1995. Oxford Science Publications.

[27] A. Kudryavtseva and V. Mazorchuk. Square Matrices as a Semigroup. http://www2.math.uu.se/ research/pub/Mazorchuk9.pdf, July 6, 2015.

[28] I. Levi. Automorphisms of normal transformation semigroups. Proceedings of the Edinburgh Mathematical Society (2) 28 (1985), 185-205.

[29] I. Levi. Automorphisms of normal partial transformation semigroups. Glasgow Mathematical Journal 29 (1987), 149-157.

[30] I. Levi. Congruences on normal transformation semigroups. Mathematica Japonica, 52 (2) (2000), $247-261$.

[31] I. Levi, D. B. McAlister, and R. B. McFadden. Groups associated with finite transformation semigroups. Semigroup Forum, 61 (3) (2000), 453-467.

[32] A. Liber. On symmetric generalized groups. Matematicheskii Sbornik N.S. 33 (75), (1953), 531-544. 
[33] A. Mal'cev. Symmetric groupoids. Matematicheskii Sbornik N.S. 31 (73), (1952), 136-151.

[34] A. Mal'cev. Multiplicative congruences of matrices. Doklady Akademii N.S. 90 (1953), 333-335.

[35] Donald B. McAlister. Semigroups generated by a group and an idempotent. Communications in Algebra, 26 (2) (1998), 515-547.

[36] P. M. Neumann. Primitive permutation groups and their section-regular partitions. Michigan Mathematical Journal 58 (2009), 309-322.

[37] B. Schein and B. Teclezghi. Endomorphisms of finite full transformation semigroups. Proceedings of the American Mathematical Society 126 (1998), no.9, 2579-2587.

[38] E. Sutov. Homomorphisms of the semigroup of all partial transformations. Izvestiya Vysshikh Uchebnykh Zavedenii. Matematika 22, no. 3, (1961), 177-184.

[39] J.S.V. Symons. Normal transformation semigroups. Journal of the Australian Mathematical Society Series A 22 (1976), no. 4, 385-390.

[40] K. Urbanik. A representation theorem for $v^{*}$-algebras. Fundamenta Mathematicae, 52 (1963), $291-317$.

(Araújo) Universidade Aberta, R. Escola Politécnica, 147, 1269-001 Lisboa, Portugal \& CEMATCiÊnCias, Departamento de Matemática, Faculdade de Ciências, Universidade de Lisboa, 1749-016, Lisboa, Portugal, Jjaraujo@fc.ul.Pt

(Bentz) School of Mathematics \& Physical Sciences, University of Hull, Hull, Hu6 7RX, UK, W.BENTZ@HULL.AC.UK

(Gomes) CEMat-CiênCias, Departamento de Matemática, Faculdade de Ciências, Universidade De Lisboa, 1749-016, Lisboa, Portugal, GmCunha@fC.ul.PT 